\title{
eXtended Hybridizable Discontinuous Galerkin with Heaviside enrichment for heat bimaterial problems
}

\author{
Ceren Gürkan · Martin Kronbichler · Sonia \\ Fernández-Méndez
}

\begin{abstract}
A novel strategy for the Hybridizable Discontinuous Galerkin (HDG) solution of heat bimaterial problems is proposed. It is based on eXtended Finite Element philosophy, together with a level set description of interfaces. Heaviside enrichment on cut elements and cut faces is used to represent discontinuities across the interface. A suitable weak form for the HDG local problem on cut elements is derived, accounting for the discontinuous enriched approximation, and weakly imposing continuity or jump conditions over the material interface. The computational mesh is not required to fit the interface, simplifying and reducing the cost of mesh generation and, in particular, avoiding continuous remeshing for evolving interfaces. Numerical experiments demonstrate that X-HDG keeps the accuracy of standard HDG methods in terms of optimal convergence and superconvergence.
\end{abstract}

Keywords interface · bimaterial · Hybridizable Discontinuous Galerkin (HDG) · highorder $\cdot$ level-sets $\cdot \mathrm{X}$-FEM $\cdot \mathrm{X}$-HDG

\section{Introduction}

Hybridizable discontinuous Galerkin (HDG) methods [4,5] are a family of discontinuous Galerkin (DG) methods that have proved their efficiency not only in the field of computational fluid dynamics $[6,22,23]$, but also in the context of elliptic and wave problems $[13,17$, $19,26]$. In addition to the advantages of most DG methods $[3,16,21,24]$ - such as intrinsic stabilization thanks to numerical fluxes or suitability for code vectorization, parallel computation and adaptivity - the hybridization process in HDG allows for a reduction in the number of degrees of freedom in the final linear system, similarly to static condensation in the context of high-order continuous finite elements, see for instance [13]. In particular, for a Laplace equation the globally coupled unknowns reduce to the approximation of the trace of the solution at the mesh skeleton, i.e., the sides (or faces in 3D) of the mesh. Moreover,

C. Gürkan and S. Fernández-Méndez

Laboratori de Càlcul Numèric (LaCàN), Universitat Politècnica de Catalunya (UPC), Barcelona, Spain.

E-mail: [ceren.gurkan, sonia.fernandez]@upc.edu

M. Kronbichler

Institute for Computational Mechanics, Technical University of Munich, Garching, Germany.

E-mail: kronbichler@lnm.mw.tum.de 
convergence of order $k+1$ in the $\mathcal{L}_{2}$ norm is proved not only for the primal unknown, but also for its derivative [4,7]. Therefore, a simple element-by-element postprocessing leads to a superconvergent approximation of the primal variables, with convergence of order $k+2$ in the $\mathcal{L}_{2}$ norm.

HDG has also shown its suitability for the solution of bimaterial problems. In $[18,25]$ HDG is applied to the solution of bimaterial Stokes and Poisson problems. However, in these algorithms the mesh needs to be adapted to properly fit the interface geometry, requiring continuous remeshing in the case of evolving interfaces. In [8] an HDG method for unfitted meshes is proposed, based on the definition of ansatz functions that are defined in the whole computational domain and represent the discontinuities over the interface. However, convergence is proved only for linear and quadratic approximation, and numerical examples show that higher order convergence may not be reached in general.

An alternative strategy for the solution of bimaterial problems, based on HDG together with eXtended Finite Element (X-FEM, see [2,9,11]) philosophy, is proposed here: the eXtended Hybridizable Discontinuous Galerkin (X-HDG) method. X-HDG was first introduced in [14] for the solution of problems with voids, and it is further developed here for the solution of bimaterial interface problems by introducing an enriched approximation for elements and faces cut by the interface in order to represent discontinuities across the interface as usual in X-FEM methods for bimaterial problems. A level set function is used for the geometrical representation of interfaces which allows for a computational mesh not fitted to the interface.

In the context of X-FEM, Heaviside enrichment is the usual choice to represent strong discontinuities along interfaces, i.e., discontinuities in the primal unknown. In the case of weak discontinuities (i.e., the unknown is continuous with a discontinuous derivative) several options have been proposed in the context of high-order continuous finite element approximations. On the one hand, several authors propose modified or corrected ridge enrichment functions that lead to approximations with a weak discontinuity on the interface, see for instance $[2,9,10]$. However, these approximations may not reach high-order convergence rates. On the other hand, Heaviside enrichment combined with a proper weak form is considered in [20], ensuring high-order convergence rates.

In the context of high-order DG methods, a Heaviside enrichment is the natural choice for both kinds of discontinuities. The Heaviside enrichment introduces a strong discontinuity on the interface that can be treated with the same strategies as the element-by-element discontinuities of the approximation. In X-HDG, Heaviside enrichment is applied to both cut elements and cut faces.

Following HDG philosophy, a trace variable is defined on the mesh skeleton (the union of the faces of the elements) that allows stating the so-called local problem in every element. The local problem expresses the solution in every element in terms of the trace variable. For elements not cut by the interface, the solution of the local problem is the standard HDG local solver. For elements cut by the interface, a modified local problem is derived, which accounts for the discontinuous enriched approximation inside the element, and for the continuity or jump conditions over the interface. A new trace variable is introduced representing the trace of the solution on the interface, which is isolated afterwards using the interface conditions. The resulting modified local solver for cut elements has the same structure as for standard elements - the solution at the element is expressed exclusively in terms of the trace variable on the mesh skeleton-but with modified matrices, in order to account for the enriched approximation and the interface conditions in the element. The problem is then closed with the so-called conservativity conditions that, after replacement of local solvers, lead to the global problem: a system of equations involving only the trace variable on the 
mesh skeleton. Given the trace, the local problem can be used to compute the solution inside the elements in a second step. Then, as in standard HDG, an X-HDG superconvergent solution can be computed through an element-by-element postprocessing algorithm that is properly modified for cut elements to take the discontinuities into account. In all computations, the robust and efficient methodology proposed in [14] is considered for numerical integration in cut elements and on cut faces. Is it based on a polynomial parametrization of the interface in each element with the same degree as the finite element approximation, ensuring high-order convergence rates.

$\mathrm{X}$-HDG inherits the advantages of X-FEM methods, i.e., the computational mesh is not required to fit the interface, simplifying and reducing the cost of mesh generation and, in particular, avoiding continuous remeshing for evolving interfaces. At the same time, the computational efficiency, stability, accuracy and optimal convergence and superconvergence of HDG is retained.

The X-HDG formulation for bimaterial problems is presented in Section 2, introducing the enriched approximation, recalling the HDG weak form of the global problem and for uncut elements, and proposing a modified local problem for cut elements. For the sake of simplicity, the formulation is detailed for a bimaterial problem, but the extension to multimaterial problems is straightforward. The ability of X-HDG to handle elements that are split by the interface into more than two regions is discussed in section 2.4. X-HDG allows considering different enrichment functions in each element in a natural way, which is not straightforward for X-FEM based on continuous approximations.

Numerical examples in section 3 demonstrate the applicability of the method, and how X-HDG keeps the accuracy, optimal convergence, and superconvergence of HDG for the solution of bimaterial interface problems.

\section{X-HDG formulation with Heaviside enrichment for bimaterial problems}

Let $\Omega \subset \mathbb{R}^{d}$ be a bounded domain divided in two disjoint subdomains

$$
\bar{\Omega}=\overline{\Omega_{1}} \cup \overline{\Omega_{2}}, \quad \Omega_{1} \cap \Omega_{2}=\emptyset
$$

with an interface

$$
\mathcal{I}=\overline{\Omega_{1}} \cap \overline{\Omega_{2}} .
$$

The following bimaterial problem is considered,

$$
\begin{aligned}
-\nabla \cdot(\nu \boldsymbol{\nabla u})=f & \text { in } \Omega_{1} \cup \Omega_{2}, \\
\llbracket u \boldsymbol{n} \rrbracket=\mathbf{0} & \text { on } \mathcal{I}, \\
\llbracket \nu \boldsymbol{n} \cdot \boldsymbol{\nabla u \rrbracket}=0 & \text { on } \mathcal{I}, \\
u=u_{D} & \text { on } \Gamma_{D}, \\
-\nu \boldsymbol{n} \cdot \boldsymbol{\nabla u}=g_{N} & \text { on } \Gamma_{N},
\end{aligned}
$$

where $u$ is the solution, $\nu$ is a material coefficient with discontinuous definition across the interface (that is, $\nu=\nu_{i}$ in $\Omega_{i}$ for $i=1,2$ ), $f$ is a given source term, $u_{D}$ are prescribed values on the Dirichlet boundary $\Gamma_{N}$, and $g_{N}$ is a prescribed flux on the Neumann boundary $\Gamma_{N}$, with $\Gamma_{D} \cup \Gamma_{N}=\partial \Omega$. The jump $\llbracket \cdot \rrbracket$ operator is defined at an interface (material interface or, later, faces between elements), using values from the domains to the left and right of the interface,

$$
\llbracket \odot \rrbracket=\odot_{L}+\odot_{R},
$$


always involving the normal vector. That is, for instance, at the material interface $\mathcal{I}$ between the subdomains $\Omega_{1}$ and $\Omega_{2}$, the jump of a vector $\boldsymbol{w}$ is $\llbracket \boldsymbol{w} \cdot \boldsymbol{n} \rrbracket:=\boldsymbol{w}_{1} \cdot \boldsymbol{n}_{1}+\boldsymbol{w}_{2} \cdot \boldsymbol{n}_{2}=$ $\left(\boldsymbol{w}_{1}-\boldsymbol{w}_{2}\right) \cdot \boldsymbol{n}_{1}$, where $\boldsymbol{n}_{i}$ is the normal vector exterior to $\Omega_{i}$, and $\boldsymbol{w}_{i}$ denotes the restriction of $\boldsymbol{w}$ to $\Omega_{i}$.

The domain $\Omega$ is now assumed to be covered by a finite element mesh with $\mathrm{n}_{\mathrm{el}}$ disjoint elements $K_{i}$, such that

$$
\bar{\Omega}=\bigcup_{i=1}^{\mathrm{n}_{\mathrm{el}}} \bar{K}_{i}, \quad K_{i} \cap K_{j}=\emptyset \text { for } i \neq j .
$$

The union of all $\mathrm{n}_{\mathrm{fc}}$ faces $\Gamma_{i}$ (sides in $2 \mathrm{D}$ ) is denoted as

$$
\Gamma:=\bigcup_{i=1}^{\mathrm{n}_{\mathrm{el}}} \partial K_{i}=\bigcup_{f=1}^{\mathrm{n}_{\mathrm{fc}}} \Gamma_{f} .
$$

In the discontinuous setting, equation (1) is expressed as a first order system with some equations local to the elements and some global equations. The local element-by-element problems correspond to the statement of the PDE in (1) in each element $K_{i}$ subject to Dirichlet boundary conditions, that is,

$$
\left.\begin{array}{rlrl}
\boldsymbol{\nabla} \cdot \boldsymbol{q}=f & & \text { in } K_{i} \\
\boldsymbol{q}+\nu \boldsymbol{\nabla} u=0 & & \text { in } K_{i} \\
u=\widehat{u} & & \text { on } \partial K_{i}
\end{array}\right\} \text { if } \mathcal{I} \cap K_{i}=\emptyset,
$$

for $i=1, \ldots, \mathrm{n}_{\mathrm{e}}$. Two new variables are introduced: $\boldsymbol{q}$ corresponding to the flux of $u$, splitting the PDE into two first order PDEs, and $\widehat{u}$ corresponding to the trace of $u$ at the mesh faces $\Gamma$. The trace $\widehat{u}$ is a single valued variable on each face, with the same value when seen from both sides of an interior face. Figure 1 shows an example of an HDG computational mesh, with elemental nodes and trace nodes. The local problems have been particularized

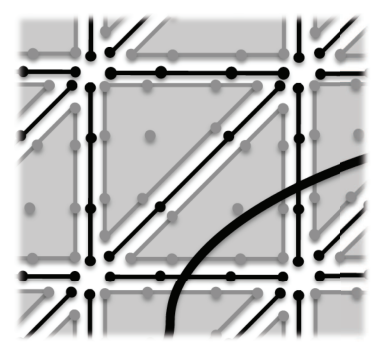

Fig. 1 Example of a third degree HDG discretization. Nodal approximation at elements (gray nodes) for $u$ and $\boldsymbol{q}$, and nodal approximation at sides (black nodes) for the trace $\widehat{u}$. Some elements and sides are cut by the interface.

for elements cut by the interior boundary (3b), including the interface conditions, and for 
standard elements (3a). Given the trace $\widehat{u}$, the local problems (3) can be solved in each element to determine the solution $u$ and the flux $\boldsymbol{q}$. Thus, the problem now reduces to determine the trace $\widehat{u}$. This is done by imposing the so-called conservativity conditions (also known as global equations), that is, the continuity of the flux across element boundaries

$$
\llbracket \boldsymbol{q} \cdot \boldsymbol{n} \rrbracket=0 \quad \text { on } \Gamma \backslash \partial \Omega,
$$

and the boundary conditions, equivalent to the respective conditions in (1),

$$
\widehat{u}=\mathbb{P}_{2}\left(u_{D}\right) \text { on } \Gamma_{D}, \quad \boldsymbol{q} \cdot \boldsymbol{n}=g_{N} \text { on } \Gamma_{N},
$$

where $\mathbb{P}_{2}\left(u_{D}\right)$ is the $\mathcal{L}^{2}$ projection of the data $u_{D}$ on the approximation space on the faces, i.e., a least-squares fitting.

It is important noting that the continuity of the solution $u$ across $\Gamma$ is imposed by the Dirichlet boundary condition in the local problems (3) and the fact that $\widehat{u}$ is single valued on $\Gamma$.

The discretization of the conservativity condition (4) and the local problems (3), with the boundary conditions (5), leads to the complete X-HDG formulation. The following discrete spaces for elemental variables, $u$ and $\boldsymbol{q}$, and for the trace variable, $\widehat{u}$, are considered

$$
\begin{aligned}
& \mathcal{V}^{h}:=\left\{v \in \mathcal{L}^{2}(\Omega):\left.v\right|_{K_{i}} \in \mathcal{P}_{k}\left(K_{i}\right) \quad \text { if } K_{i} \cap \mathcal{I}=\emptyset,\right. \\
& \left.\left.v\right|_{K_{i}} \in \mathcal{P}_{k}\left(K_{i}\right) \oplus H \mathcal{P}_{k}\left(K_{i}\right) \text { if } K_{i} \cap \mathcal{I} \neq \emptyset\right\}, \\
& \Lambda^{h}:=\left\{\hat{v} \in \mathcal{L}^{2}(\Gamma):\left.\hat{v}\right|_{\Gamma_{i}} \in \mathcal{P}_{k}\left(\Gamma_{i}\right) \quad \text { if } \Gamma_{i} \cap \mathcal{I}=\emptyset,\right. \\
& \left.\left.\hat{v}\right|_{\Gamma_{i}} \in \mathcal{P}_{k}\left(\Gamma_{i}\right) \oplus H \mathcal{P}_{k}\left(\Gamma_{i}\right) \text { if } \Gamma_{i} \cap \mathcal{I} \neq \emptyset\right\},
\end{aligned}
$$

where $\mathcal{P}_{k}$ denotes the space of polynomials of degree less or equal to $k . H$ is a Heaviside function enriching the approximation in cut elements and on cut faces, which can be defined, for instance, as

$$
H=\left\{\begin{aligned}
1 & \text { in } \Omega_{1} \\
-1 & \text { in } \Omega_{2}
\end{aligned}\right.
$$

to introduce discontinuities across the interface $\mathcal{I}$.

Remark 1 In X-HDG the approximation space is enriched with a Heaviside function and, therefore, the approximations are discontinuous across the interface for both the solution $u$ and the flux $\boldsymbol{q}$. This is a natural choice for enriching the flux $\boldsymbol{q}$, whose tangential component is discontinuous across the interface. For the solution $u$, even though it is continuous, $\mathrm{X}-\mathrm{HDG}$ considers a discontinuous approximation to keep the discontinuous setting of HDG also across the interface. Continuity of the solution $u$ and the normal flux $\boldsymbol{q} \cdot \boldsymbol{n}$ across the interface is imposed weakly, as usually done across interior faces in HDG. In fact, the X-HDG method proposed here is formally equivalent to a standard HDG method applied on a cut mesh combining triangular and quadrilateral elements with a $\mathcal{P}_{k}$ polynomial approximation (i.e., a complete $k$-th degree polynomial basis also for quadrilaterals), but organized and implemented in an alternative way to keep the original triangular computational mesh and the original unknown structure, as usual in X-FEM methods. Thus, X-HDG keeps the superconvergence and stability properties of standard HDG, but in accordance with an X-FEM philosophy.

Remark 2 For the sake of simplicity, all derivations in this section assume that elements are split into two regions only, which corresponds to the usual situation. Thus, one Heaviside enrichment is considered, see (6). However, with high-order approximations more complicated situations, dividing elements or faces into more regions, may appear, see section 2.4 
and the numerical example in section 3.5. In such a case, an enrichment with multiple Heaviside functions is proposed in section 2.4 to ensure an independent approximation in each region of the element or face.

Remark 3 The description of the interface can be done with a level set function as usual in $\mathrm{X}$-FEM methods, see for instance [14]. The level set function is given by its nodal values on the computational mesh using an $r$-th degree approximation. In most numerical tests a $k$-th degree level set function is used for the X-HDG solution and an additional level set function with polynomial degree $k+1$ is used for the postprocessed solution. Nevertheless, for examples with evolving interfaces and more involved interface geometries an accurate representation of the interface is crucial to keep the X-HDG accuracy, see the kidney-shaped interface example in section 3.4. According to the analysis for standard HDG with meshes fitted to the interface in [18], a level set function with degree $r=2 k+1$ ensures optimal convergence of X-HDG in the more general situation.

The next sections present the details of the X-HDG formulation, stating the discretization of the local problems for standard and cut elements and the discretization of the conservativity condition. The local problem at elements not cut by the interface (3a) and the global problem (4) are discretized as usual in HDG [5,4] and recalled in sections 2.1 and 2.3. The discretization of the local problem for cut elements ( $3 b$ ) is developed in section 2.2.

To simplify the presentation, in an abuse of notation, the same notation is used for the numerical approximation, belonging to the finite dimensional spaces (6), and the exact solution, that is $u, \boldsymbol{q}$ and $\widehat{u}$.

\subsection{Local problem for standard elements}

This section recalls the standard HDG local problem on an element $K_{i}$ not cut by the interface. It corresponds to the discretization of (3a), that is: given $\widehat{u} \in \Lambda^{h}$, find $u \in \mathcal{P}_{k}\left(K_{i}\right)$, $\boldsymbol{q} \in\left[\mathcal{P}_{k}\left(K_{i}\right)\right]^{d}$ such that

$$
\begin{array}{cl}
\int_{K_{i}} v \boldsymbol{\nabla} \cdot \boldsymbol{q} d V+\int_{\partial K_{i}} \tau \nu v(u-\widehat{u}) d S=\int_{K_{i}} v f d V & \forall v \in \mathcal{P}_{k}\left(K_{i}\right) \\
\int_{K_{i}} \frac{1}{\nu} \boldsymbol{q} \cdot \boldsymbol{w} d V-\int_{K_{i}} u \boldsymbol{\nabla} \cdot \boldsymbol{w} d V+\int_{\partial K_{i}} \widehat{u} \boldsymbol{w} \cdot \boldsymbol{n} d S=0 & \forall \boldsymbol{w} \in\left[\mathcal{P}_{k}\left(K_{i}\right)\right]^{d} .
\end{array}
$$

The first equation in (7) can be derived from the first equation in (3a) by applying integration by parts, replacing the flux by the numerical flux

$$
\widehat{\boldsymbol{q}}:=\boldsymbol{q}+\tau \nu(u-\widehat{u}) \boldsymbol{n},
$$

and undoing the integration by parts. The second equation is obtained from the weak form of the second equation in (3a), applying integration by parts and replacing the boundary condition $u=\widehat{u}$ on the element boundary.

Remark 4 The parameter $\tau$ in the definition of the numerical flux (8) is a non-negative stabilization parameter usually taken of order $\mathcal{O}(1)$. For each element, it may be taken as a positive constant on all faces, or positive on one arbitrary face and zero at the rest (single face). Both options lead to stable and optimally convergent solutions, with superconvergent postprocessed solutions. See for instance $[12,4]$ for details on the influence of this parameter on the solution behavior. 
The discretization of the local problem (7) leads to a system of equations of the form

$$
\begin{aligned}
& \mathbf{A}_{u u}^{K_{i}} \mathbf{u}^{i}+\mathbf{A}_{u q}^{K_{i}} \mathbf{q}^{i}+\mathbf{A}_{u \widehat{u}}^{K_{i}} \boldsymbol{\Lambda}^{i}=\mathbf{f}_{u}^{K_{i}}, \\
& \mathbf{A}_{q u}^{K_{i}} \mathbf{u}^{i}+\mathbf{A}_{q q}^{K_{i}} \mathbf{q}^{i}+\mathbf{A}_{q \widehat{u}}^{K_{i}} \mathbf{\Lambda}^{i}=\mathbf{0},
\end{aligned}
$$

where $\mathbf{u}^{i}$ and $\mathbf{q}^{i}$ are the vectors of nodal values of $u$ and $\boldsymbol{q}$ in element $K_{i}$. The vector $\boldsymbol{\Lambda}^{i}$ is the vector of nodal values of $\widehat{u}$ on the $n$ faces of the element ( $n=3$ for triangles and $n=4$ for tetrahedra),

$$
\boldsymbol{\Lambda}^{i}:=\left[\begin{array}{c}
\widehat{\mathbf{u}}^{\mathbf{F}_{i 1}} \\
\vdots \\
\widehat{\mathbf{u}}^{i n}
\end{array}\right],
$$

where $\widehat{\mathbf{u}}^{f}$ denotes the nodal values of $\widehat{u}$ on face $\Gamma_{f}$, and $\mathbf{F}_{i j}$ is the number of the $j$-th face of element $K_{i}$. Note that the subindices in the matrices A refer to the space for the weighting function and the admissible solution, respectively.

System (9) can be solved for $\mathbf{u}^{i}$ and $\mathbf{q}^{i}$ in each element, obtaining the so-called local solver in the element $K_{i}$,

$$
\mathbf{u}^{i}=\mathbf{U}^{K_{i}} \boldsymbol{\Lambda}^{i}+\mathbf{f}_{U}^{K_{i}}, \quad \mathbf{q}^{i}=\mathbf{Q}^{K_{i}} \boldsymbol{\Lambda}^{i}+\mathbf{f}_{Q}^{K_{i}},
$$

with the following matrices and vectors

$$
\begin{aligned}
& {\left[\begin{array}{c}
\mathbf{U}^{K_{i}} \\
\mathbf{Q}^{K_{i}}
\end{array}\right]=-\mathbb{A}^{-1}\left[\begin{array}{l}
\mathbf{A}_{u \widehat{u}}^{K_{i}} \\
\mathbf{A}_{q \widehat{u}}^{K_{i}}
\end{array}\right],\left[\begin{array}{c}
\mathbf{f}_{U}^{K_{i}} \\
\mathbf{f}_{Q}^{K_{i}}
\end{array}\right]=\mathbb{A}^{-1}\left[\begin{array}{c}
\mathbf{f}_{u}^{K_{i}} \\
\mathbf{0}
\end{array}\right],} \\
& \mathbb{A}=\left[\begin{array}{ll}
\mathbf{A}_{u u}^{K_{i}} & \mathbf{A}_{u q}^{K_{i}} \\
\mathbf{A}_{q u}^{K_{i}} & \mathbf{A}_{q q}^{K_{i}}
\end{array}\right] .
\end{aligned}
$$

In other words, the values of the solution $\mathbf{u}^{i}$ and $\mathbf{q}^{i}$ on element $i$ can be explicitly expressed in terms of the trace on its faces, $\boldsymbol{\Lambda}^{i}$.

\subsection{Local problem for cut elements}

The X-HDG local problem on an element $K_{i}$ cut by the interface corresponds to the discretization of (3b), that is: given $\widehat{u} \in \Lambda^{h}$, find $u \in \mathcal{P}_{k}\left(K_{i}\right) \oplus H \mathcal{P}_{k}\left(K_{i}\right), \boldsymbol{q} \in\left[\mathcal{P}_{k}\left(K_{i}\right) \oplus\right.$ $\left.H \mathcal{P}_{k}\left(K_{i}\right)\right]^{d}$ and $\widetilde{u}^{i} \in \mathcal{P}_{k}\left(I_{i}\right)$ such that

$$
\begin{gathered}
\int_{K_{i}} v \boldsymbol{\nabla} \cdot \boldsymbol{q} d V+\int_{\partial K_{i}} \tau \nu v(u-\widehat{u}) d S+2 \int_{\mathcal{I}_{i}} \tau\left\{\nu v\left(u-\widetilde{u}^{i}\right)\right\} d S=\int_{K_{i}} v f d V \\
\int_{K_{i}} \frac{1}{\nu} \boldsymbol{q} \cdot \boldsymbol{w} d V-\int_{K_{i}} u \boldsymbol{\nabla} \cdot \boldsymbol{w} d V+\int_{\partial K_{i}} \widehat{u} \boldsymbol{w} \cdot \boldsymbol{n} d S+\int_{\mathcal{I}_{i}} \widetilde{u}^{i} \llbracket \boldsymbol{w} \cdot \boldsymbol{n} \rrbracket d S=0
\end{gathered}
$$

for all $v \in \mathcal{P}_{k}\left(K_{i}\right) \oplus H \mathcal{P}_{k}\left(K_{i}\right)$ and $\boldsymbol{w} \in\left[\mathcal{P}_{k}\left(K_{i}\right) \oplus H \mathcal{P}_{k}\left(K_{i}\right)\right]^{d}$, and

$$
\int_{\mathcal{I}_{i}} \widetilde{v} \llbracket \boldsymbol{q} \cdot \boldsymbol{n} \rrbracket d S+2 \tau \int_{\mathcal{I}_{i}} \widetilde{v}\left(\{\nu u\}-\{\nu\} \widetilde{u}^{i}\right) d S=0 \quad \forall \widetilde{v} \in \mathcal{P}_{k}\left(\mathcal{I}_{i}\right) .
$$


Note that the jump in $\nu, v$ and $u$ across $\mathcal{I}_{i}:=K_{i} \cap \mathcal{I}$ is taken into account by jump and mean operations. The mean operator $\{\cdot\}$ is defined as taking values from the left and right domains sharing the interface, in this case

$$
\{\odot\}=\frac{1}{2}\left(\odot_{1}+\odot_{2}\right) .
$$

A new trace variable $\widetilde{u}^{i} \in \mathcal{P}_{k}\left(I_{i}\right)$ is introduced in the local equations (13), which approximates the trace of the solution on the interface $\mathcal{I}_{i}$. With the new trace variable $\widetilde{u}^{i}$, the local equations (13) can be derived following the standard HDG procedure for each one of the domains, $K_{i} \cap \Omega_{1}$ and $K_{i} \cap \Omega_{2}$, and summing the obtained weak forms. Compared to the weak form for standard elements (7), the local equations for a cut element (13) have two additional terms corresponding to integrals along the interface $\mathcal{I}_{i}$. They involve the new trace variable $\widetilde{u}^{i}$ and take the discontinuous nature of the approximation space into account. These new terms weakly impose the condition $u=\widetilde{u}^{i}$ on $\mathcal{I}_{i}$, ensuring weak continuity of $u$ across the interface. The local problem is closed with (14), that is, the weak form of the conservativity condition across the interface, $\llbracket \widehat{\boldsymbol{q}} \cdot \boldsymbol{n} \rrbracket=0$ on $\mathcal{I}_{i}$, considering the numerical flux defined in (8), i.e., $\widehat{\boldsymbol{q}} \cdot \boldsymbol{n}=\boldsymbol{q} \cdot \boldsymbol{n}+\tau\left(u-\widetilde{u}^{i}\right)$.

Remark 5 The aproximation space $\mathcal{P}_{k}\left(I_{i}\right)$ is represented by a one-dimensional polynomial nodal basis of degree $k$ on the 1D reference element $(-1,1)$, which is mapped to a nodal basis on an approximation of the interface $\mathcal{I}_{i}$. The mapping is done with an $r$-th degree parametrization given by $r+1$ points on the interface. The $r$-th degree parametrization of the interface is also used for the definition of numerical quadrature schemes within the cut element, accounting for the discontinuous nature of the approximation across the interface, see $[2,14]$ for details, and an example with degree $r=3$ of the level set function in Figure 2 . With the proposed approach, the integrals on both sides of the interface are computed separately.
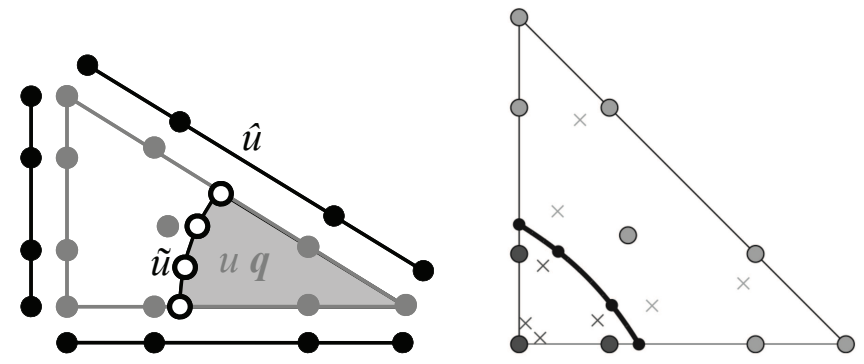

Fig. 2 Representation of the X-HDG discretization in an element cut by the interface (left): the elemental variables $u$ and $\boldsymbol{q}$ are depicted by gray nodes, the trace variable $\widehat{u}$ by black nodes, and the trace on the interface $\widetilde{u}^{i}$ by circles. Representation of the reference element for an element cut by the interface (right): interface representation and numerical quadrature with cubic parametrization of the interface. The color of the nodes indicates the sign of the level set function defining the interface. Crosses are exemplary integration points.

The discretization of the local equations (13) leads to a system of equations of the form

$$
\begin{aligned}
{\left[\mathbf{A}_{u u}^{K_{i}}+\mathbf{A}_{u u}^{\mathcal{I}_{i}}\right] \mathbf{u}^{i}+\mathbf{A}_{u q}^{K_{i}} \mathbf{q}^{i}+\mathbf{A}_{u \widehat{u}}^{K_{i}} \mathbf{\Lambda}^{i}+\mathbf{A}_{u \widetilde{u}^{i}}^{\mathcal{I}_{i}} \widetilde{\mathbf{u}}^{i} } & =\mathbf{f}_{u}^{K_{i}} \\
\mathbf{A}_{q u}^{K_{i}} \mathbf{u}^{i}+\mathbf{A}_{q q}^{K_{i}} \mathbf{q}^{i}+\mathbf{A}_{q \widehat{u}}^{K_{i}} \mathbf{\Lambda}^{i}+\mathbf{A}_{q \widetilde{u}^{i}}^{\mathcal{I}_{i}} \widetilde{\mathbf{u}}^{i} & =\mathbf{0}
\end{aligned},
$$


similar to the system for standard elements (9), but with three new matrices corresponding to integrals on the interface $\mathcal{I}_{i}$ (marked with the superindex $\mathcal{I}_{i}$ ), and the nodal values for the new trace variable, $\widetilde{\mathbf{u}}^{i}$.

On other hand, the discretization of the conservativity condition (14) leads to a discrete equation of the form

$$
\mathbf{A}_{\widetilde{u}^{i} u}^{\mathcal{I}_{i}} \mathbf{u}^{i}+\mathbf{A}_{\widetilde{u}^{i} q}^{\mathcal{I}_{i}} \mathbf{q}^{i}+\mathbf{A}_{\widetilde{u}^{i} \widetilde{u}^{i}}^{\mathcal{I}_{i}} \widetilde{\mathbf{u}}^{i}=0
$$

which allows expressing the new trace values $\widetilde{\mathbf{u}}^{i}$ in terms of the elemental values

$$
\widetilde{\mathbf{u}}^{i}=\mathbf{T}_{u}^{i} \mathbf{u}^{i}+\mathbf{T}_{q}^{i} \mathbf{q}^{i}
$$

with $\mathbf{T}_{u}^{i}=-\left[\mathbf{A}_{\widetilde{u}^{i} \widetilde{u}^{i}}^{\mathcal{I}_{i}}\right]^{-1} \mathbf{A}_{\widetilde{u}^{i} u}^{\mathcal{I}_{i}}, \mathbf{T}_{q}^{i}=-\left[\mathbf{A}_{\widetilde{u}^{i} \widetilde{u}^{i}}^{\mathcal{I}_{i}}\right]^{-1} \mathbf{A}_{\widetilde{u}^{i} q}^{\mathcal{I}_{i}}$. This expression corresponds to a Schur complement for eliminating the trace solution $\widetilde{u}^{i}$. This elimination of a trace variable $\widetilde{u}^{i}$ in favor of the local variables, $u$ and $q$, is logically the opposite elimination as the one expressed by the local solver. Substituting $\widetilde{\mathbf{u}}^{i}$ in (16) by (17) leads to the final discrete local problem

$$
\begin{gathered}
{\left[\mathbf{A}_{u u}^{K_{i}}+\mathbf{A}_{u u}^{\mathcal{I}_{i}}+\mathbf{A}_{u \widetilde{u}^{i}}^{\mathcal{I}_{i}} \mathbf{T}_{u}^{i}\right] \mathbf{u}^{i}+\left[\mathbf{A}_{u q}^{K_{i}}+\mathbf{A}_{u \widetilde{u}^{i}}^{\mathcal{I}_{i}} \mathbf{T}_{q}^{i}\right] \mathbf{q}^{i}+\mathbf{A}_{u \widehat{u}}^{K_{i}} \mathbf{\Lambda}^{i}=\mathbf{f}_{u}^{K_{i}}} \\
{\left[\mathbf{A}_{q u}^{K_{i}}+\mathbf{A}_{q \widetilde{u}^{i}}^{\mathcal{I}_{i}} \mathbf{T}_{u}^{i}\right] \mathbf{u}^{i}+\left[\mathbf{A}_{q q}^{K_{i}}+\mathbf{A}_{q \widetilde{u}^{i}}^{\mathcal{I}_{i}} \mathbf{T}_{q}^{i}\right] \mathbf{q}^{i}+\mathbf{A}_{q \widehat{u}}^{K_{i}} \mathbf{\Lambda}^{i}=\mathbf{0} .}
\end{gathered}
$$

Now, system (18) can be solved for $\mathbf{u}^{i}$ and $\mathbf{q}^{i}$, obtaining the local solver in the cut element $K_{i}$, i.e., equation (11) with (12) and

$$
\mathbb{A}=\left[\begin{array}{cc}
{\left[\mathbf{A}_{u u}^{K_{i}}+\mathbf{A}_{u u}^{\mathcal{I}_{i}}+\mathbf{A}_{u \widetilde{u}^{i}}^{\mathcal{I}_{i}} \mathbf{T}_{u}^{i}\right]} & {\left[\mathbf{A}_{u q}^{K_{i}}+\mathbf{A}_{u \widetilde{u}^{i}}^{\mathcal{I}_{i}} \mathbf{T}_{q}^{i}\right]} \\
{\left[\mathbf{A}_{q u}^{K_{i}}+\mathbf{A}_{q \widetilde{u}^{i}}^{\mathcal{I}_{i}} \mathbf{T}_{u}^{i}\right]} & {\left[\mathbf{A}_{q q}^{K_{i}}+\mathbf{A}_{q \widetilde{u}^{i}}^{\mathcal{I}_{i}} \mathbf{T}_{q}^{i}\right]}
\end{array}\right] .
$$

The structure of the local solver is exactly the same as for non-cut elements (11), thanks to the fact that the internal trace variable $\widetilde{u}^{i}$ has been expressed in terms of the local variables. Note, however, that the size of the matrices involved in the local solver is different for a standard element and for a cut element due to the enriched approximation. In the simplest case (see Remark 2), there are twice as many degrees of freedom for cut elements and faces.

Remark 6 The extension of the formulation to other conditions on the interface is straightforward. As an example, consider a problem with non-homogeneous conditions on the interface, $u_{2}-u_{1}=\alpha$ and $\llbracket-\nu \boldsymbol{n} \cdot \nabla u \rrbracket=g$ on $\mathcal{I}_{i}$, where $u_{i}$ denotes the restriction of function $u$ to $\Omega_{i}$. Then, defining $\widetilde{u}^{i}$ as the trace of $u_{1}$ (i.e., $u_{1}=\widetilde{u}^{i}$ on $\mathcal{I}_{i}$ ), we have $u_{2}=\widetilde{u}^{i}+\alpha$ on $\mathcal{I}_{i}$, and therefore, new terms $\int_{\mathcal{I}_{i}} \tau \nu_{2} v_{2} \alpha$ and $-\int_{\mathcal{I}_{i}} \alpha \boldsymbol{w}_{2} \cdot \boldsymbol{n}_{2}$ appear in the right hand side of the first and second equation in (13), respectively, and the right hand side of (14) becomes $\int_{\mathcal{I}_{i}} \widetilde{v} g d S+\int_{\mathcal{I}_{i}} \tau \nu_{2} \widetilde{v} \alpha d S$

\subsection{Global problem}

The local problem, both in a standard element or in a cut element, leads to the local solver (11) that expresses the solution in the element, $u$ and $\boldsymbol{q}$, in terms of the trace values at its boundary, $\widehat{u}$. Thus, the problem is reduced to determine the trace nodal values $\left\{\widehat{\mathbf{u}}^{f}\right\}_{f=1}^{n_{f c}}$ on the mesh skeleton $\Gamma$. For this purpose the so-called global problem is stated, which corresponds to the discretization of the conservativity condition on $\Gamma$ (4), with the boundary conditions (5), replacing $\boldsymbol{q}$ by the numerical flux (8). 
The weak form for the global problem is: find $\widehat{u} \in \Lambda^{h}$ such that $\widehat{u}=\mathbb{P}_{2}\left(u_{D}\right)$ on $\Gamma_{D}$ and

$$
\int_{\Gamma} \widehat{v} \llbracket \boldsymbol{q} \cdot \boldsymbol{n} \rrbracket d S+2 \tau \int_{\Gamma} \widehat{v}(\{\nu u\}-\{\nu\} \widehat{u}) d S=\int_{\Gamma_{N}} \widehat{v} g_{N} d S
$$

for all $\widehat{v} \in \Lambda^{h}$ with $\widehat{v}=0$ on $\Gamma_{D}$. In this weak form the definitions of the jump (2) and mean (15) operators are extended for exterior faces, here on the Neumann boundary $\Gamma_{N}$, considering only the value from the unique element containing the face; that is $\llbracket \odot \rrbracket=\odot$ and $\{\odot\}=\frac{1}{2} \odot$ on $\Gamma_{N} \subset \Gamma$.

Remark 7 The Dirichlet boundary conditions of (5) could alternatively have been implemented by setting the nodal values for $\widehat{u}$ on the faces on the Dirichlet boundary by interpolation. However, an $\mathcal{L}^{2}$ projection of the prescribed values has to be considered to ensure a superconvergence of $u^{*}$, see Remark 9. Otherwise, the average $\int_{K_{i}} u d V$ may not converge with order $k+2$, and $u^{*}$ may not reach the full rate of convergence $k+2$.

As usual in HDG, the discretization of (19) for every face $\Gamma_{f}$ leads to an equation of the form

$$
\mathbf{A}_{\widehat{u} u}^{f, L} \mathbf{u}^{L(f)}+\mathbf{A}_{\widehat{u} q}^{f, L} \mathbf{q}^{L(f)}+\mathbf{A}_{\widehat{u} u}^{f, R} \mathbf{u}^{R(f)}(f)+\mathbf{A}_{\widehat{u} q}^{f, R} \mathbf{q}^{R(f)}+\mathbf{A}_{\widehat{u} \widehat{u}}^{f} \widehat{\mathbf{u}}^{f}=\mathbf{g}^{f} .
$$

Inserting the expressions of the local solver (11) for the elements $K_{L(f)}$ and $K_{R(f)}$ in (20) for every face $\Gamma_{f}$ leads to a system of equations involving only the trace variables $\left\{\widehat{\mathbf{u}}^{f}\right\}_{f=1}^{\mathrm{n}_{\mathrm{fc}}}$.

As usual in an HDG code, the implementation of the method involves a loop over elements. For each element, the matrices and vectors for the local solver (11) are computed, and the contribution to the equation (20) is assembled for each face of the element. Once the system is assembled for all elements and Dirichlet boundary conditions are imposed according to Remark 7, the system can be solved. Then, given the trace variables $\left\{\widehat{\mathbf{u}}^{f}\right\}_{f=1}^{\mathrm{n}_{\mathrm{fc}}}$, the solution, $\mathbf{u}^{i}$ and $\boldsymbol{q}^{i}$, can be computed for each element using (11).

It is important noting that X-HDG keeps the structure of a standard HDG code. The main differences are: (i) the modified local problem for cut elements (16), and the corresponding matrices in the local solver, (ii) the modified numerical integration in cut elements and on cut faces, (iii) the increased number of degrees of freedom for the enriched approximation in cut elements and on cut faces, which has to be taken into account also for the assembly of the matrices involving cut faces.

Remark 8 Compared to standard HDG, the size of the system of equations to be solved for X-HDG is larger because the number of degrees of freedom for cut faces doubles as compared to a standard case (in the simplest case, see Remark 2). However, in practical applications cut faces are usually a small portion of the whole set of faces.

Remark 9 Similarly to standard HDG, a second element-by-element postprocessing can be done to compute an X-HDG superconvergent solution. For a standard element, $K_{i} \cap \mathcal{I}=\emptyset$, the superconvergent approximation is computed as in standard HDG: find $u^{*} \in \mathcal{P}_{k+1}\left(K_{i}\right)$ such that

$$
\begin{gathered}
\int_{K_{i}} \nu \nabla u^{*} \cdot \nabla v d V=-\int_{K_{i}} \boldsymbol{q} \cdot \boldsymbol{\nabla} v d V \quad \forall v \in \mathcal{P}_{k+1}\left(K_{i}\right), \\
\int_{K_{i}} u^{*} d V=\int_{K_{i}} u d V
\end{gathered}
$$


For an element cut by the interface, $K_{i} \cap \mathcal{I} \neq \emptyset$, the superconvergent approximation is computed finding $u^{*} \in \mathcal{P}_{k+1}\left(K_{i}\right) \oplus H \mathcal{P}_{k+1}\left(K_{i}\right)$ such that

$$
\begin{gathered}
\int_{K_{i}} \nu \nabla u^{*} \cdot \nabla v d V=-\int_{K_{i}} \boldsymbol{q} \cdot \nabla v d V \quad \forall v \in \mathcal{P}_{k+1}\left(K_{i}\right) \oplus H \mathcal{P}_{k+1}\left(K_{i}\right), \\
\int_{K_{i}} u^{*} d V=\int_{K_{i}} u d V \quad \text { and } \quad \int_{K_{i}} H u^{*} d V=\int_{K_{i}} H u d V .
\end{gathered}
$$

The solution of this element-by-element computation, $u^{*}$, converges with order $k+2$ in the $\mathcal{L}_{2}$ norm. See $[4,7]$ for details and other possible computations of a superconvergent solution.

\subsection{Cut element split into more than two regions}

As mentioned in Remark 2, in the most general situation some elements and faces may be split by the interface into more than two regions, requiring an enrichment with several Heaviside functions. For instance, for a triangle split into three regions, as shown in Figure 3, an

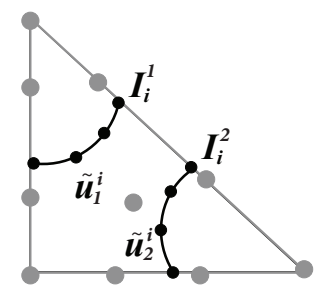

Fig. 3 Triangle split by the interface into 3 regions, with two elemental interfaces $\mathcal{I}_{i}^{1}$ and $\mathcal{I}_{i}^{2}$, with corresponding auxiliary trace variables $\widetilde{u}_{1}^{i}$ and $\widetilde{u}_{2}^{i}$. The approximation is enriched with two Heaviside functions.

approximation with two Heaviside functions should be considered to properly represent the discontinuity. The local problem in this case is: find $u \in \mathcal{P}_{k}\left(K_{i}\right) \oplus H^{1} \mathcal{P}_{k}\left(K_{i}\right) \oplus H^{2} \mathcal{P}_{k}\left(K_{i}\right)$, $\boldsymbol{q} \in\left[\mathcal{P}_{k}\left(K_{i}\right) \oplus H^{1} \mathcal{P}_{k}\left(K_{i}\right) \oplus H^{2} \mathcal{P}_{k}\left(K_{i}\right)\right]^{d}$ such that

$$
\begin{aligned}
& \int_{K_{i}} v \boldsymbol{\nabla} \cdot \boldsymbol{q} d V+ \int_{\partial K_{i}} \tau \nu v(u-\widehat{u}) d S \\
&+2 \int_{\mathcal{I}_{i}^{1}} \tau\left\{\nu v\left(u-\widetilde{u}_{1}^{i}\right)\right\} d S+2 \int_{\mathcal{I}_{i}^{2}} \tau\left\{\nu v\left(u-\widetilde{u}_{2}^{i}\right)\right\} d S=\int_{K_{i}} v f d V \\
& \int_{K_{i}} \frac{1}{\nu} \boldsymbol{q} \cdot \boldsymbol{w} d V-\int_{K_{i}} u \boldsymbol{\nabla} \cdot \boldsymbol{w} d V+\int_{\partial K_{i}} \widehat{u} \boldsymbol{w} \cdot \boldsymbol{n} d S+\int_{\mathcal{I}_{i}^{1}} \widetilde{u}_{1}^{i} \llbracket \boldsymbol{w} \cdot \boldsymbol{n} \rrbracket d S+\int_{\mathcal{I}_{i}^{2}} \widetilde{u}_{2}^{i} \llbracket \boldsymbol{w} \cdot \boldsymbol{n} \rrbracket d S=0
\end{aligned}
$$
for all $v \in \mathcal{P}_{k}\left(K_{i}\right) \oplus H^{1} \mathcal{P}_{k}\left(K_{i}\right) \oplus H^{2} \mathcal{P}_{k}\left(K_{i}\right)$ and $\boldsymbol{w} \in\left[\mathcal{P}_{k}\left(K_{i}\right) \oplus H^{1} \mathcal{P}_{k}\left(K_{i}\right) \oplus H^{2} \mathcal{P}_{k}\left(K_{i}\right)\right]^{d}$, where $\mathcal{I}_{i}^{1}$ and $\mathcal{I}_{i}^{2}$ are the interfaces splitting the element into three regions, $H^{1}$ and $H^{2}$ are Heaviside functions to represent the discontinuities on both interfaces, and $\widetilde{u}_{1}^{i}$ and $\widetilde{u}_{2}^{i}$ are new trace variables approximating the trace of the solution on $\mathcal{I}_{i}^{1}$ and $\mathcal{I}_{i}^{2}$, respectively. Following the rationale in section 2.2, the trace variables $\widetilde{u}_{1}^{i}$ and $\widetilde{u}_{2}^{i}$ are expressed in terms of $u$ and $\boldsymbol{q}$ using the weak form of the conservativity condition (14) on each interface. Inserting them into the local problem again leads to a local solver with the same structure as (11), expressing $u$ and $\boldsymbol{q}$ in terms of the global trace variable $\widehat{u}$. 
Obviously, at faces that are split by the interface into more than two regions, an enrichment with several Heaviside functions must also be considered for $\widehat{u}$. A numerical example showing the performance of X-HDG when some elements are cut twice by the interface can be found in section 3.5.

We note that in a DG framework the use of different enrichment functions in neighboring elements does not imply any difficulty. In X-HDG the continuity between elements is imposed in a weak form, giving total freedom for a decoupled definition of the approximation space in every element. This is not the case in the context of continuous finite elements. In X-FEM, the usual practice is considering a unique definition of the level set functions in the whole domain, to ensure continuity of the approximation in a simple way. For illustration purposes, Figure 4 shows a discretization example with 2 triangles cut by an interface, for a continuous approximation (left) and an HDG approximation (right). In this situation, the X-HDG discretization considers two Heaviside functions for enrichment in element $K_{1}$ and the cut side, and one Heaviside function for enrichment in element $K_{2}$. Continuity is imposed weakly by the X-HDG formulation in a natural and straight-forward way. However, a standard X-FEM approximation would consider a unique Heaviside function associated to the interface in both elements, in order to keep the continuity of the approximation on the side shared by the triangles. This is because in X-FEM all nodes have to be enriched with the same enrichment function. This would lead to an artificial link between the solution in the the two separated regions in $K_{1}$, with the consequent decrease of accuracy. An alternative
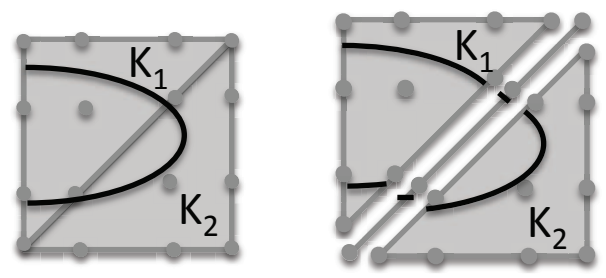

Fig. 4 Representation of 2 triangles cut by one interface for X-FEM (continuous) and X-HDG. Element $K_{1}$ is split by the interface into 3 regions. The X-HDG discretization considers 2 Heaviside functions for enrichment in element $K_{1}$ and the cut side, and 1 Heaviside in element $K_{2}$.

in the context of continuous finite element is the so-called Virtual Node Algorithm (VNA) [1], which is based on a suitable duplication of nodes and elements, instead of the classical enrichment. However, the implementation of the VNA for complicated interfaces, as the one depicted in Figure 4, may be cumbersome.

\section{Numerical examples}

The performance of novel X-HDG method is tested by several numerical examples in this section. Both straight and curved interfaces with discontinuous or continuous solutions across the interface are considered. For verification of the results obtained with X-HDG, results are compared against a standard HDG setting where the computational mesh fits the interface. An example with cut elements that are split by the interface into more than two regions is also considered, demonstrating the capability of X-HDG to handle this kind of situations. Finally the applicability of X-HDG to problems with moving interfaces, avoiding continuous remeshing to fit the interface, is shown with a numerical test. 
3.1 Straight interface with zero jump conditions

In this first test case, the Poisson equation (1) is solved over a square domain $\Omega=(-1,1)^{2}$ with a straight interface $\mathcal{I}$ at $x=0.2031$. The material parameter $\nu$ is defined as $\nu_{1}=1$ in $\Omega_{1}$ and $\nu_{2}=2.5$ in $\Omega_{2}$. In Figure 5, the linear interface $\mathcal{I}$ separating the two material domains can be seen for both the X-HDG and the HDG settings. For X-HDG the interface is represented by the level set function $\varphi(x, y)=x-0.2031$, and it is not fitted to the computational mesh. Elements cut by the interface are depicted in light gray. On other hand, for HDG the interface needs to coincide with element boundaries of the computational mesh. Note that generating a computational mesh fitting the interface is simple in this example, but may be cumbersome and costly for irregularly shaped curved interfaces, specially for evolving interfaces.
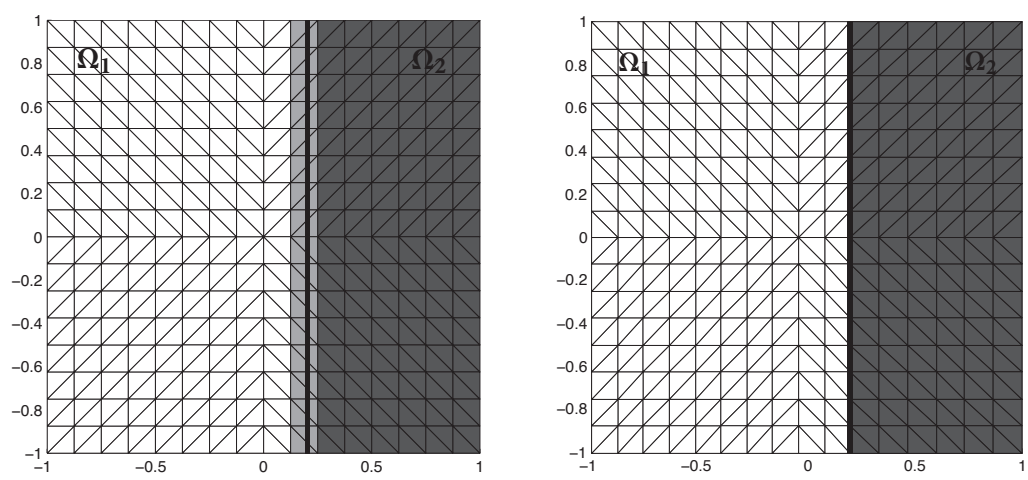

Fig. 5 Linear interface with zero jump conditions: computational mesh for X-HDG and for standard HDG after two mesh refinements. On the left, the X-HDG mesh not adapted to the linear interface boundary $\mathcal{I}$ which shown in black. Elements in white are standard elements in domain one whereas elements in dark gray are standard elements in domain two. Elements cut by the interface $\mathcal{I}$ are shown in light gray shade. On the right, HDG mesh fitting to the linear interface is shown. Elements in domain one are shown in white whereas elements in domain two are shown in dark gray.
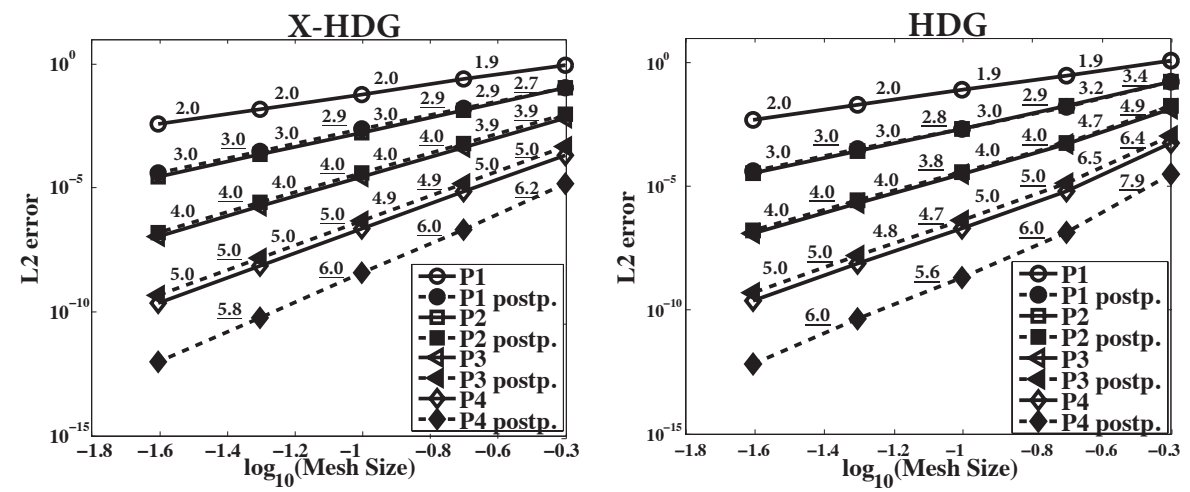

Fig. 6 Linear interface with zero jump conditions: convergence history of X-HDG and HDG with solid line for the solution $u(\mathrm{Pk})$ and dashed line for the postprocessed superconvergent solution $u^{*}$ (Pk postp). Slopes for each segment are shown. 
The source term and Dirichlet boundary conditions are set such that the analytical solution is

$$
u(x)= \begin{cases}5 x^{5} & \text { in } \Omega_{1}, \\ 2 x^{5}+A & \text { in } \Omega_{2},\end{cases}
$$

with $A=3(0.2031)^{5}$. Zero jump conditions across the interface are imposed.

Figure 6 shows the convergence of X-HDG and HDG for the bimaterial problem with straight interface. Starting with an initial mesh using four elements per coordinate direction, four mesh refinement steps are considered-each refinement doubling the number of elements per direction-both for X-HDG and HDG. The meshes for HDG do not have uniform mesh size in order to fit the interface. The characteristic mesh size in the convergence plots is the mesh size of the uniform mesh with the same number of elements. The approximation degree is varied from $k=1$ to $k=4$. The results confirm that the X-HDG strategy retains the convergence rates and accuracy of standard HDG without the need of mesh adaptation to the interface. Optimal convergence of order $k+1$ for $u$, and $k+2$ for the postprocessed superconvergent solution $u^{*}$, is observed, with a slight effect of rounding errors in the solution with the finer mesh and degree $k=4$.

\subsection{Straight interface with non-zero jump conditions}

As a second example, X-HDG is tested with a discontinuous solution over the interface, see Remark 6. The domain is again $\Omega=(0,1)^{2}$, the level set function describing the interface is $\varphi(x, y)=x-0.4$, the viscosity parameter is set to $\nu=1$ in the entire domain, and homogeneous Dirichlet boundary conditions are imposed on the boundary. The jump conditions

$$
u_{2}-u_{1}=1, \quad \llbracket \boldsymbol{n} \cdot \nabla u \rrbracket=0 \quad \text { on } \mathcal{I},
$$

are imposed on the interface, and the source term is chosen such that the analytical solution reads

$$
u(x)=\left\{\begin{array}{lr}
\sin (\pi x) \sin (\pi y) \quad \text { in } \Omega_{1}, \\
\sin (\pi x) \sin (\pi y)+1 & \text { in } \Omega_{2} .
\end{array}\right.
$$

Figure 7 shows the coarsest mesh used in our X-HDG calculations as well as the analytical solution. The mesh is refined three times-doubling the number of elements per direction in each refinement-with varying polynomial degrees between $k=2$ and $k=4$ for the convergence studies presented in Figure 8. Again, the results are compared against a conventional HDG setting where the interface is fitted by the computational mesh.

Figure 8 clearly shows that X-HDG performs equally well as the standard HDG method with a discontinuous solution over the interface without the need for a matching computational mesh.

3.3 Circular interface with zero jump conditions: heat distribution over a steady state bimaterial plate

In this example a circular interface $\mathcal{I}$ with radius $R=0.5$ is considered to divide the square domain $\Omega=(-1,1)^{2}$ into two regions, $\Omega_{1}$ and $\Omega_{2}$, as depicted in Figure 9 . The heat distribution over a plate, which is made of two materials with different thermal conductivities, is 

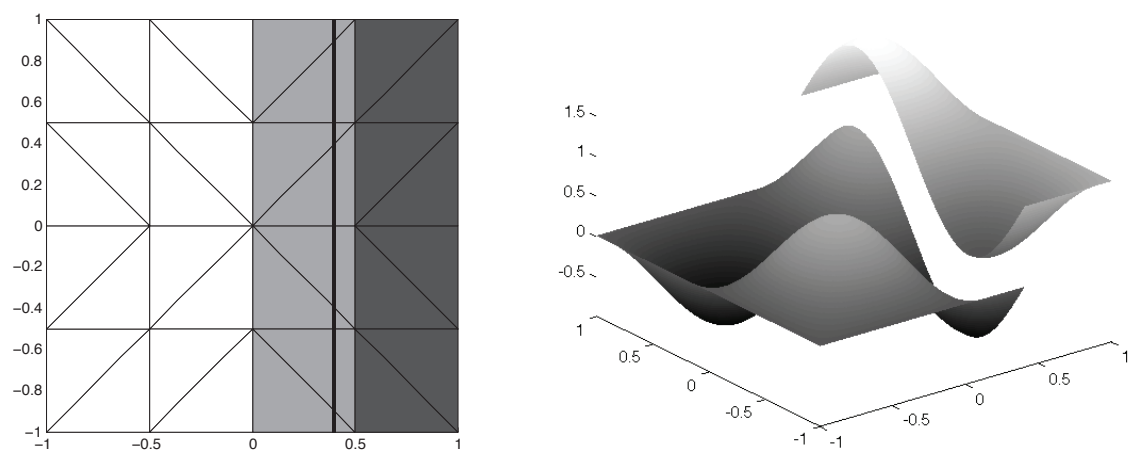

Fig. 7 Straight Interface with non-zero jump conditions: coarsest X-HDG mesh and the interface $\mathcal{I}$ (left) and analytical solution (right).
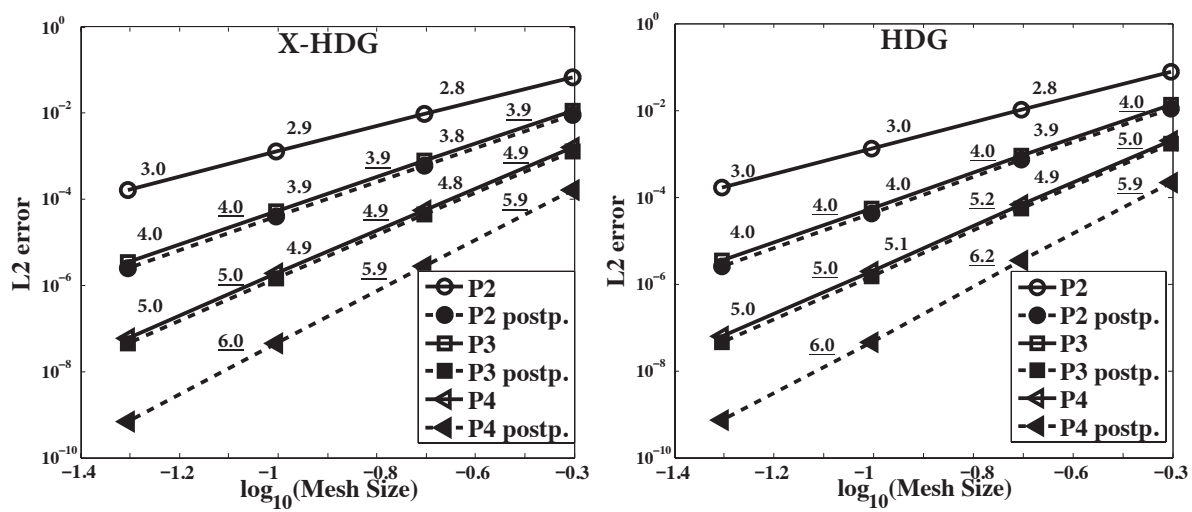

Fig. 8 Straight Interface with non-zero jump conditions: convergence history of X-HDG and HDG with solid line for the solution $u(\mathrm{Pk})$ and dashed line for the postprocessed superconvergent solution $u^{*}$ (Pk postp). Slopes for each segment are shown.

computed. The analytical solution and the thermal conductivities are defined by

$$
u(x)=\left\{\begin{array}{ll}
\frac{1}{\nu_{1}}\left(x^{2}+y^{2}\right)^{5 / 2} & \text { in } \Omega_{1}, \\
\frac{1}{\nu_{2}}\left(x^{2}+y^{2}\right)^{5 / 2}+\left(\frac{1}{\nu_{1}}-\frac{1}{\nu_{2}}\right) R^{5} & \text { in } \Omega_{2},
\end{array} \quad \text { and } \quad \nu= \begin{cases}1 & \text { in } \Omega_{1}, \\
100 & \text { in } \Omega_{2},\end{cases}\right.
$$

the corresponding source term is $f=-25\left(x^{2}+y^{2}\right)^{3 / 2}$, Dirichlet boundary conditions are set on the boundary, and zero jump conditions are set on the interface.

Figure 9 shows the mesh used in X-HDG calculations after the first refinement step as well as the analytical solution. The mesh is isotropically refined three times and the degree of approximation is varied between $k=1$ and $k=3$. Convergence plots for the X-HDG solution are shown in Figure 10. Again optimal convergence rates are reached, for both the solution $u$ and the superconvergence postprocess $u^{*}$, except for the last mesh with degree $k=3$.

This convergence loss is the result of bad-cut situations (see Figures 11 and 12, left panels) causing an ill-conditioned matrix for the system of the global problem. By a bad-cut situation we mean a case where the area ratio of two material domains of a cut element is less than 0.1. This situation is familiar from X-FEM applications and there are plenty 

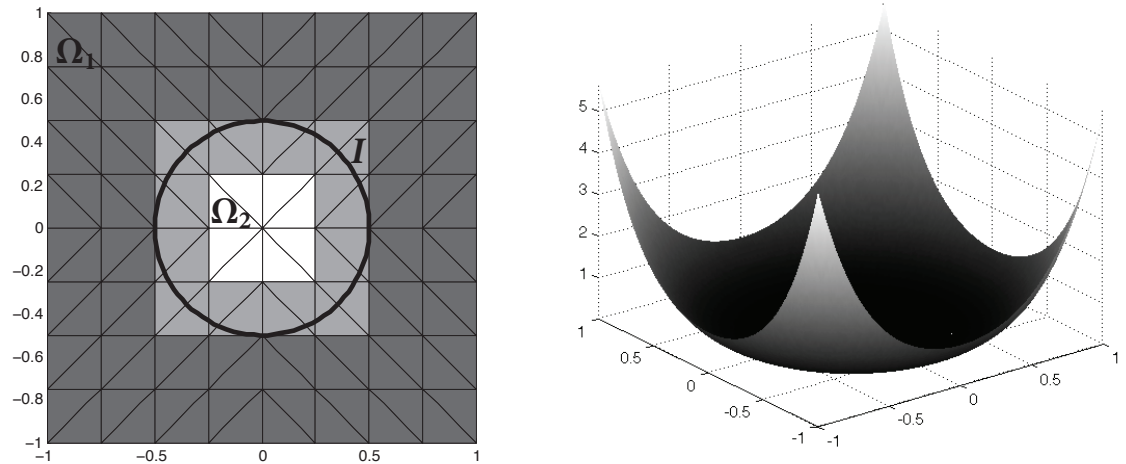

Fig. 9 Circular interface with zero jump conditions with $\nu_{1}=1$ and $\nu_{2}=100$ : mesh used in X-HDG calculations after one mesh refinement and the circular interface $\mathcal{I}$ (left), analytical solution (right).

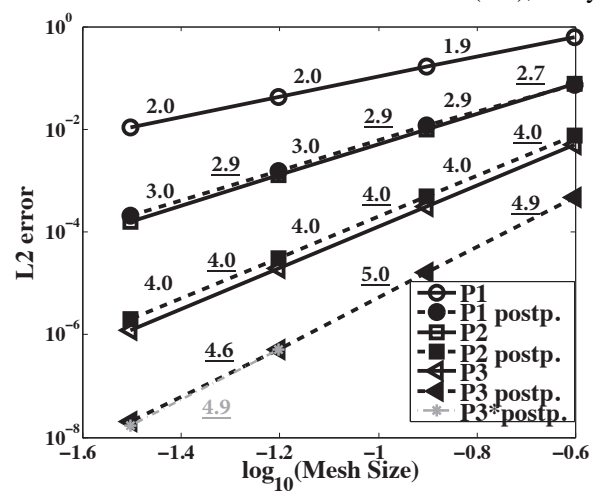

Fig. 10 Circular interface with zero jump conditions $\nu_{1}=1$ and $\nu_{2}=100$ : convergence history for the solution $u$ (Pk, solid line), the superconvergent solution $u^{*}$ (Pk postp., dashed line), and for $u^{*}$ with a slightly modified mesh to avoid ill-conditioning ( $\mathrm{P}^{*}$ postp.)

of strategies, complex or rather simple, to get rid of this ill-conditioning problem, see for instance [15]. Since the derivation of such stabilization strategies is not within the scope of this work, we choose to simply slightly modify the mesh, moving the nodes wherever we detect a bad-cut situation. Figures 11 and 12 show a zoom of the modification of the last mesh in the convergence plot for degree $k=3$. The condition number of the matrix for the completely uniform mesh is $k_{2} \simeq 10^{14}$, leading to poor accuracy in the results. After the slight modification of the mesh, avoiding the bad-cut situations, the condition number is $k_{2} \simeq 510^{9}$. The slight modification of the mesh clearly improves the conditioning and recovers optimal convergence, with rates of order $k+1$ and $k+2$ for the solution and the post processed solution, respectively. Note that this node-moving strategy is not proposed as a general solution but rather to demonstrate that X-HDG can converge optimally.

This problem was solved in [18] using standard HDG with a mesh fitting the circular interface, using superparametric elements for the curved elements along the interface. In this work, optimal convergence and similar levels of accuracy are obtained with X-HDG and standard isoparametric approximations, even in the presence of the curved elements in Figure 12. It is also worth mentioning that the analytical solution for this problem has a singularity in the fifth derivative and, therefore, theoretical convergence rates are available 

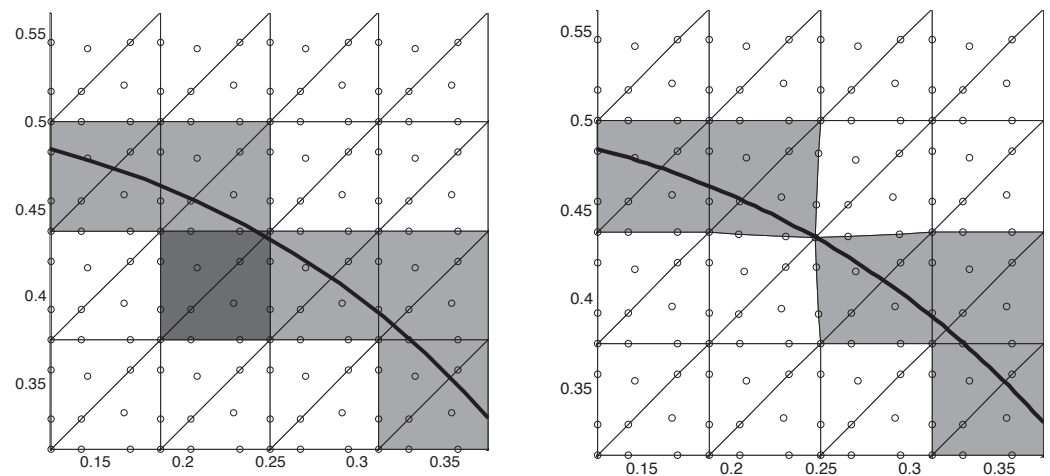

Fig. 11 Example of bad-cut situation for the circular interface: zoom of the original uniform mesh (left) and of the modified mesh (right). Standard elements are shown in white, cut elements in gray and bad-cut elements in dark gray. In this case, the vertex that is too close to the interface is moved to be placed on to top on it. The nodes of the affected elements are relocated keeping straigth sides.
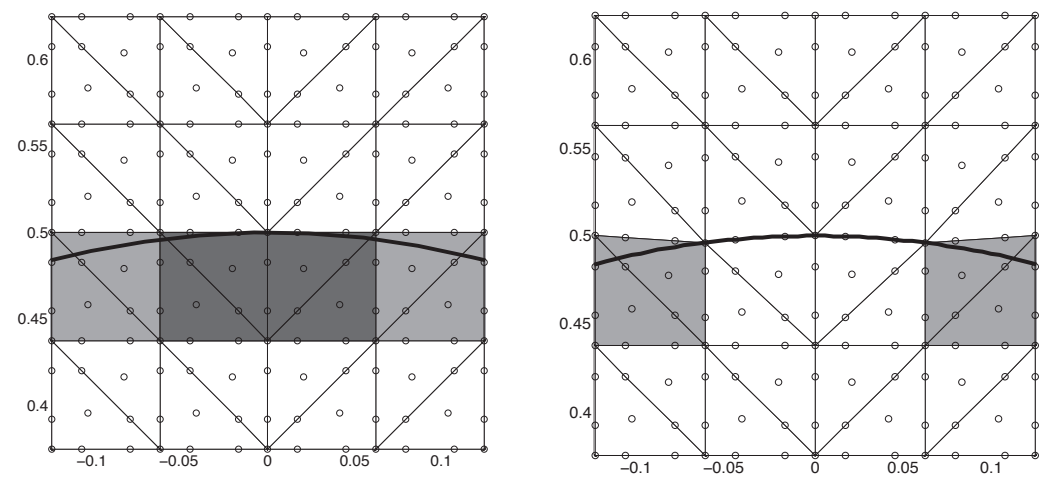

Fig. 12 Example of bad-cut situation for the circular interface: zoom of the original uniform mesh (left) and of the modified mesh (right). Standard elements are shown in white, cut elements in gray and bad-cut elements in dark gray. Again, the vertexes that are too close to the interface are moved to be placed on to top on it. If a face has two moved vertexes, the whole face is moved to a curved face fitting the interface.

up to degree $k=3$. Numerical experiments show that the convergence rate for degree $k=4$ is lower than 5 .

Figure 13 shows the convergence history and the analytical solution in case of switching the materials in $\Omega_{1}$ and $\Omega_{2}$, i.e., setting $\nu_{1}=100$ and $\nu_{2}=1$ and keeping the problem statement the same. The mesh fixing strategy is also used in this computations in the presence of bad-cut situations, again leading to optimal convergence rates.

\subsection{Kidney-shaped interface}

To study the behavior of X-HDG in the context of more involved interfaces, a problem with a kidney-shaped interface according to [18] is considered next. The domain is $\Omega=(-1,1)^{2}$, the level set function is defined as

$$
\varphi(x, y)=\left(3\left((x+0.5)^{2}+y^{2}\right)-x-0.5\right)^{2}-\left((x+0.5)^{2}+y^{2}\right)+0.1
$$



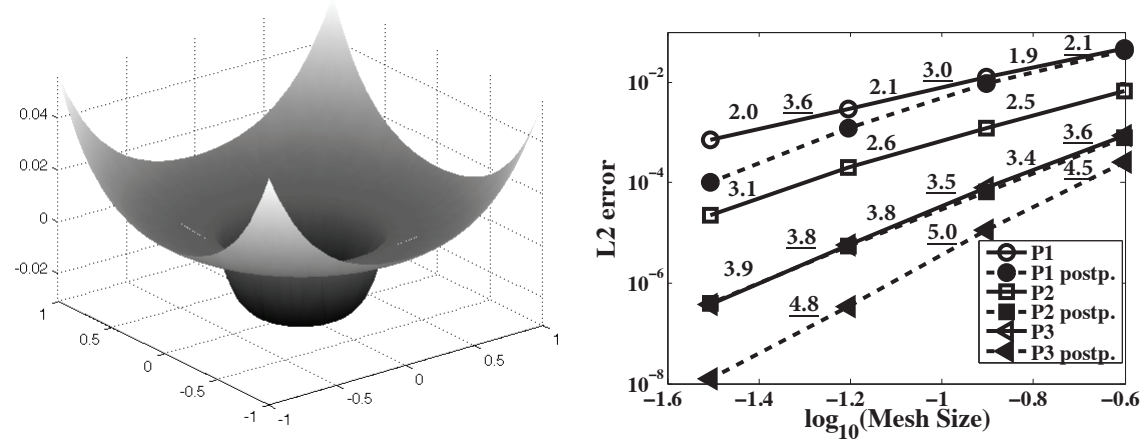

Fig. 13 Circular interface with zero jump conditions with $\nu_{1}=100$ and $\nu_{2}=1$ : analytical solution (left), convergence history for the solution $u$ (Pk, solid line) and the superconvergent solution $u^{*}$ (Pk postp., dashed line) (right).

and the material parameters are $\nu_{1}=10$ in $\Omega_{1}=\{(x, y) \in \Omega \mid \varphi(x, y)>0\}$ (i.e., the outer subdomain) and $\nu_{2}=1$ in $\Omega_{2}=\Omega \backslash \overline{\Omega_{1}}$. The source term, the Dirichlet boundary conditions, and the jump conditions on the interface are set such that the analytical solution is

$$
u(x, y)= \begin{cases}\frac{1}{\nu_{1}} \cos \left(1-x^{2}-y^{2}\right) & \text { for }(x, y) \in \Omega_{1} \\ \sin \left(2 x^{2}+y^{2}+2\right)+x & \text { for }(x, y) \in \Omega_{2}\end{cases}
$$

Figure 14 shows the convergence plots for varying approximation degree $k$ and mesh size $h$. The left panel shows the X-HDG solution with a $k$-th degree level set function and with degree $k+1$ for the superconvergent solution, whereas the right panel shows the XHDG solution with a level set function of degree $k+2$. In this example, the geometrical description of the interface crucially influences the accuracy of the solution, and a representation with degree $k+2$ is required to reach optimal convergence. For coarse meshes or low degrees, the $k$-th degree interface representation induces substantial errors in the solution. Nevertheless, it is worth noting that for finer meshes these errors become less relevant and the accuracy of an interface representation of degrees $k$ and $k+2$, respectively, is similar. For the last mesh and highest degree of approximation the effect of ill-conditioning is observed, limiting the accuracy to errors around $10^{-8}$.

The need of an accurate representation of the interface to reach optimal convergence rates is in accordance with the analysis in [18], where the authors claim that for standard HDG with a mesh fitted to the interface, superparametric elements with a geometry description of degree $2 k+1$ are necessary to ensure optimal convergence rates for the solution and for superconvergent postprocessing. The natural extension of this result to X-HDG is the need of a level set function of degree $2 k+1$ to ensure optimal convergence in the general case. Nonetheless, in this example a geometrical description of the interface of degree $k+2$ is enough to obtain maximum accuracy for each mesh and degree.

\subsection{Rectangular interface with double enrichment}

The heat equation is solved over a rectangular plate $\Omega=(0,10) \times(0,1.5)$, with an approximation of degree 3 . The plate is composed of two different materials separated by a rectangular interface. The subdomain inside the rectangular interface is $\Omega_{2}=(0,7) \times(0.65,0.85)$, 

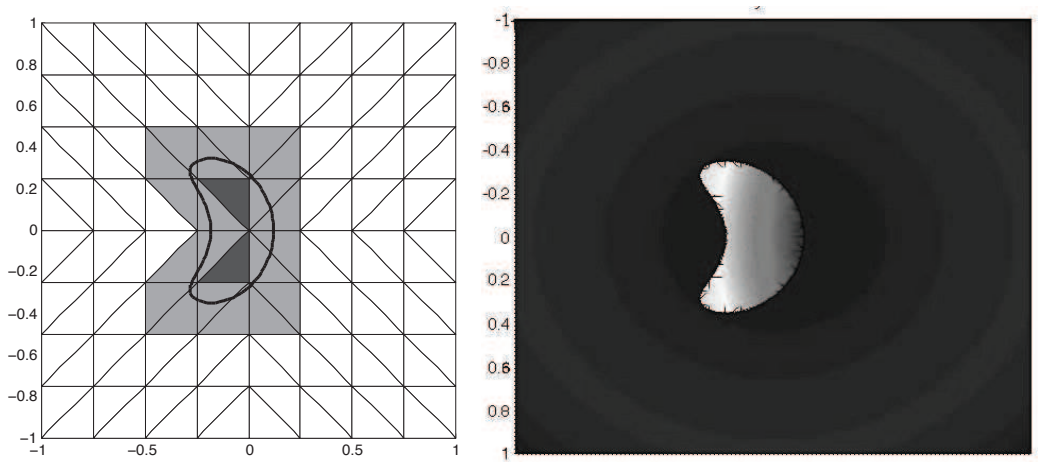

Fig. 14 Kidney-shaped interface: mesh after two refinements together with the kidney shaped interface (left) and solution (right).
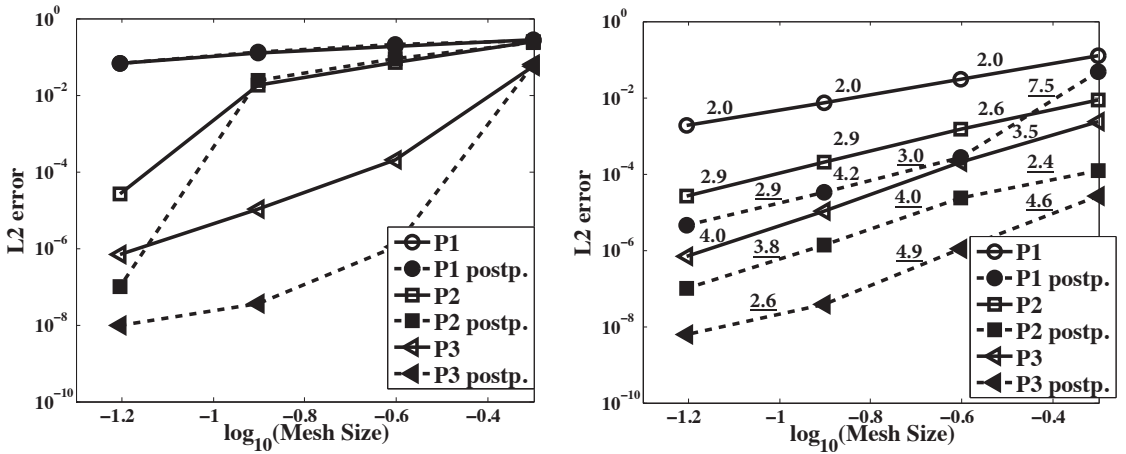

Fig. 15 Kidney interface: convergence plots with a level set function of degree $k+1$ (left) and of degree $k+2$ (right).

with permittivity constant $\nu_{2}=1$. The remainder of the domain is $\Omega_{1}=\Omega \backslash \Omega_{2}$ with permittivity constant $\nu_{1}=10$, see Figure 16 . Homogenous Neumann boundary conditions are imposed at $\{x=0\}$ and $\{x=10\}$, whereas at $\{y=0\}$ and at $\{y=1.5\}$ Dirichlet boundary conditions are set to 0 and 1 , respectively.

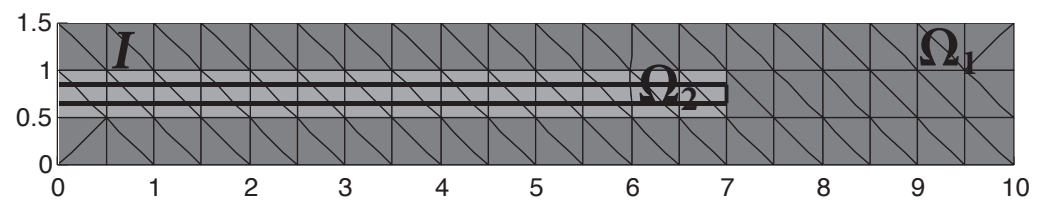

Fig. 16 Rectangular Interface: domain and computational mesh. Elements cut by the interface are shown in light gray.

Considering the computational mesh shown in Figure 16, the elements cut by the interface (in light gray) are split into three regions, two with material constant $\nu=\nu_{1}$, and the interior one with $\nu=\nu_{2}$. In this situation, cut elements and cut faces should be enriched 


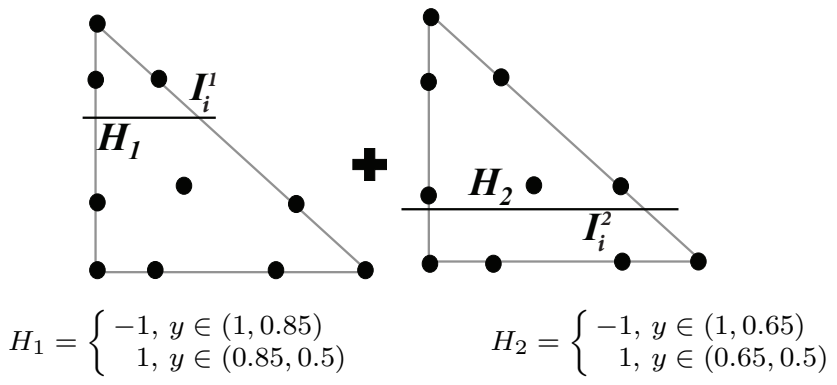

Fig. 17 Rectangular Interface: representation of the two Heaviside functions used for the double enrichment at cut elements.
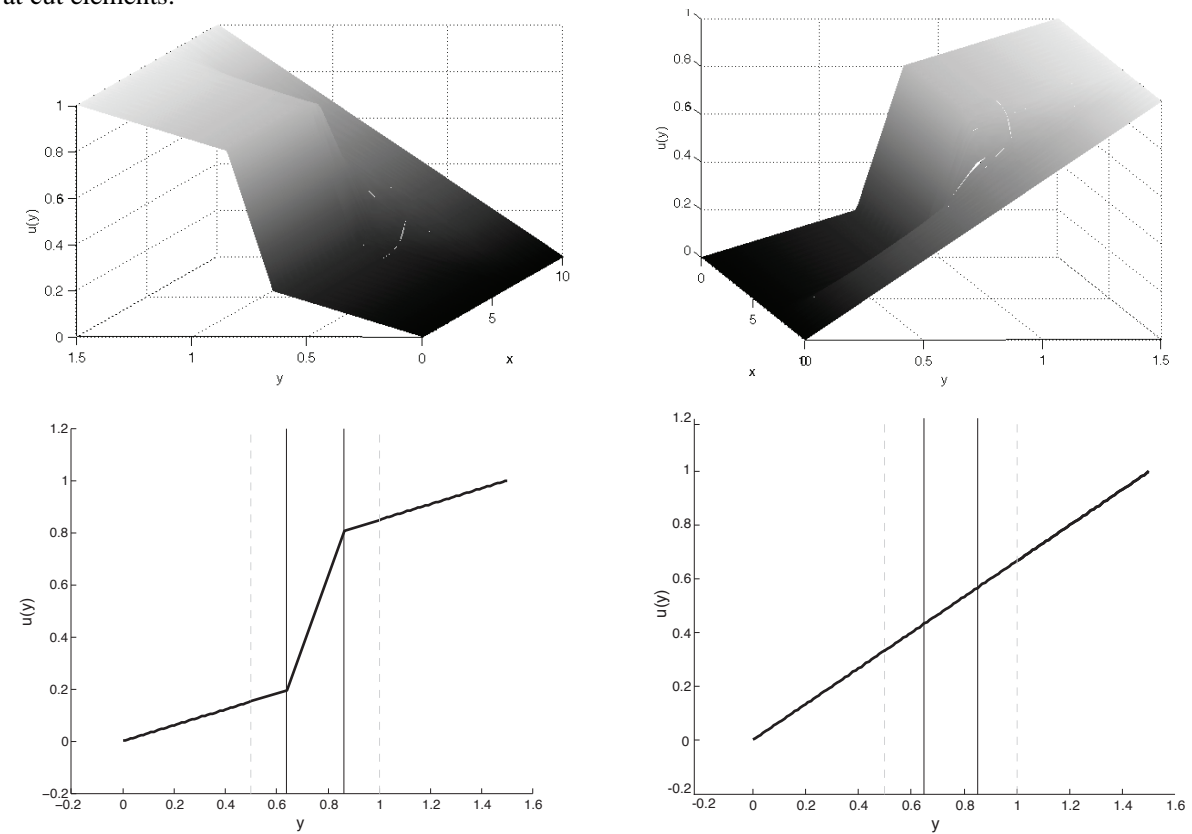

Fig. 18 Rectangular Interface: X-HDG solution, with degree $k=3$ and double enrichment at cut elements and cut faces, from two different points of view (top), and sections on $\{x=0\}$ (bottom left) and on $\{x=$ $10\}$ (bottom right). Vertical discontinuous and continuous lines represent element boundaries and interfaces, respectively.

with two Heaviside functions, as explained in section 2.4, to have a completely independent approximation for the solution in the three regions. That is, in the cut elements the solution is approximated as

$$
u \simeq a+H_{1} b+H_{2} c
$$

where $a, b$ and $c$ are polynomials of degree 3 , and $H_{1}$ and $H_{2}$ are the two Heaviside functions represented in Figure 17. Faces cut by the rectangular interface are also enriched with these two Heaviside functions, and the face at $y=7$, shared by a cut element and a standard element, is not enriched. 
Figure 18 shows the X-HDG solution from different viewing angles and over two cross sections. By using double enrichment in the cut elements, the approximations in the three regions are independent, and the X-HDG solution can freely adjust to the kinks in the analytical solution. The solution is verified to match with an HDG solution in a fine mesh adapted to the interface. As expected, on the right boundary (Neumann boundary $\{x=10\}$ with constant permittivity parameter $\nu=\nu_{1}$, far enough to the material interface) the solution is close to a linear variation from temperature 0 to temperature 1 . On the left boundary (Neumann boundary $\{x=0\}$ with two materials) the solution is close to a linear variation in the three regions, with larger slope for the interior region, which has smaller permittivity $\nu=\nu_{2}<\nu_{1}$. In the interior of the domain the solution varies in a continuous way between this two sections, with sharper variations close to $x=7$.

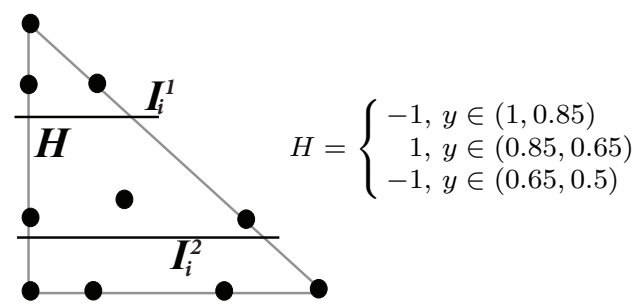

Fig. 19 Rectangular Interface: Heaviside function for the single enrichment solution.
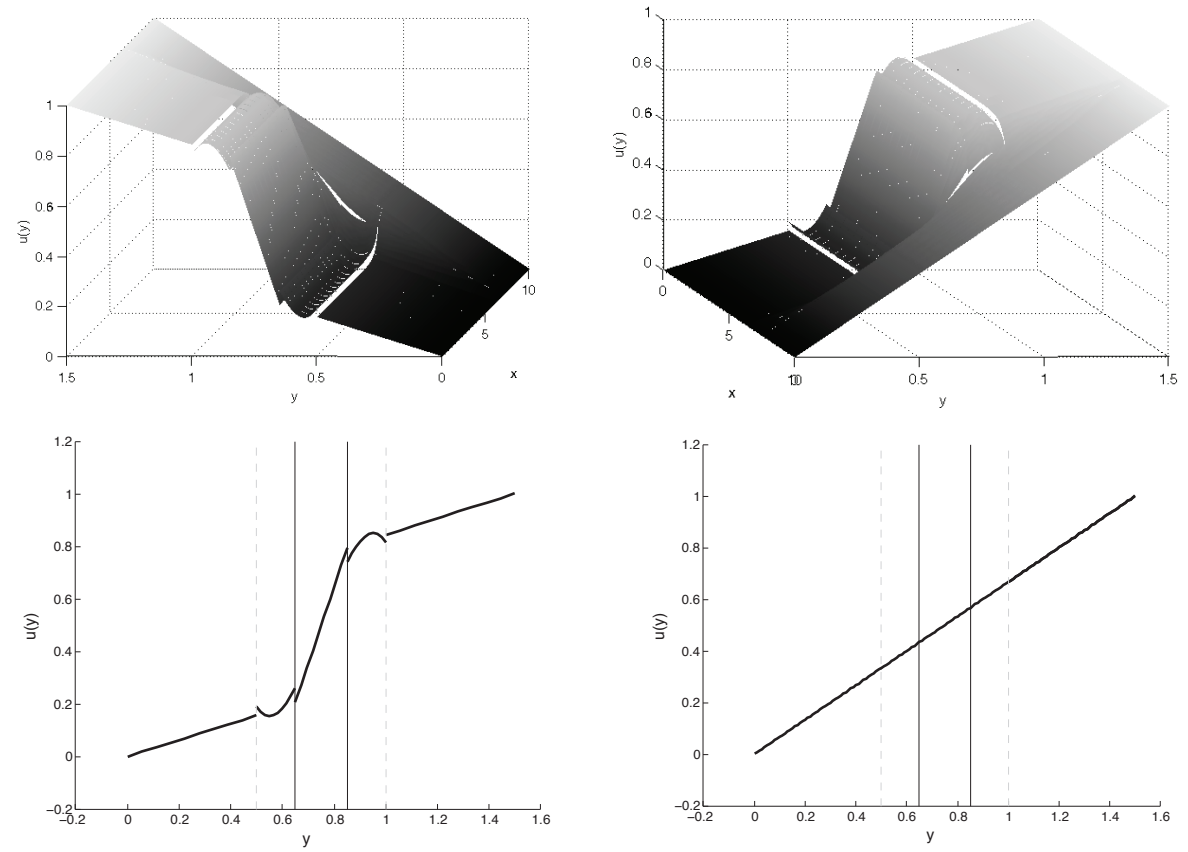

Fig. 20 Rectangular Interface: X-HDG solution, with degree $k=3$ and single enrichment at cut elements and cut faces, from two different points of view (top), and sections on $\{x=0\}$ (bottom left) and on $\{x=$ $10\}$ (bottom right). Vertical discontinuous and continuous lines represent element boundaries and interfaces, respectively. The approximation is not rich enough to capture the solution. 
To verify the convenience of considering two Heaviside enrichments in the cut elements, the problem is now solved with a single Heaviside function. That is, the approximation at cut elements is

$$
u \simeq a+H b
$$

with the Heaviside function $H$ represented in Figure 19. This would be the standard approach for an X-FEM formulation based on continuous finite elements: a single enrichment function is defined in the whole domain, and for this example, this means a single Heaviside enrichment in the cut elements.

Figure 20 shows the X-HDG solution with single enrichment, again from different viewing angles and over two cross sections. The section on $\{x=0\}$ shows how the single enrichment is not suitable to capture the solution, due to the non-physical dependency of the approximation on the top $(y>0.85)$ and bottom $(y<0.65)$ regions. This causes overand undershoots close to the interface, and large discontinuties at the element boundaries, demonstrating that the approximation with a single enrichment is not rich enough to properly adjust to the kinks in the solution.
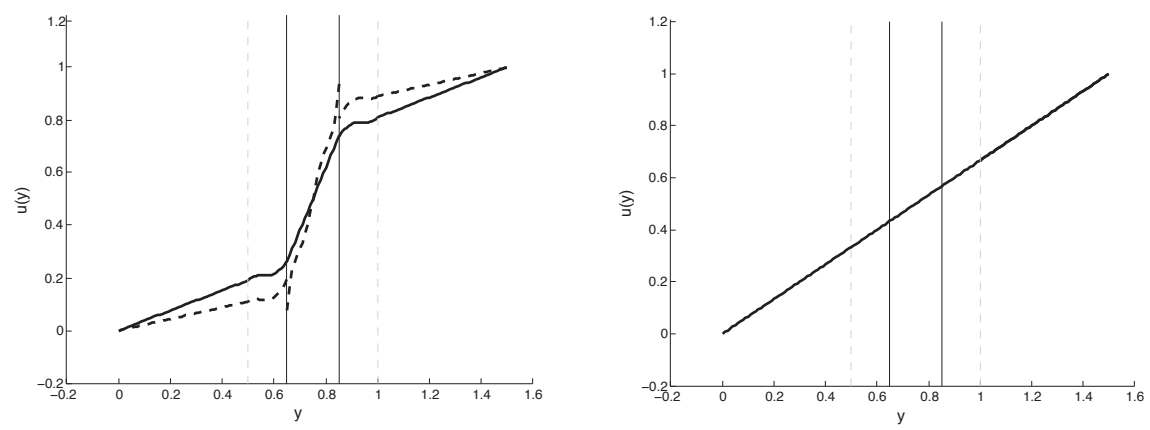

Fig. 21 Rectangular Interface: sections of the X-FEM solution, with degree $k=3$, on $\{x=0\}$ (left) and on $\{x=10\}$ (right), for $\beta=100$ (dashed line) and $\beta=1000$ (solid line). Vertical discontinuous and continuous lines represent element boundaries and interfaces, respectively.

To corroborate this conclusion, the problem is also solved with an X-FEM formulation based on continuous finite elements, with a unique Heaviside function defined in the whole domain to represent the interface, that is, with the single enrichment at cut elements. Nitsche's method (or, equivalently, the interior penalty method) is considered for weakly imposing the continuity of the solution and of the normal flux across the interface, see [20] for details. Similarly to the X-HDG solution with single enrichment, the X-FEM solution is not able to properly represent the solution. Figure 21 shows the section on $\{x=0\}$ for two different values of the Nitsche parameter $\beta$. For $\beta=100$ the X-FEM solution presents large discontinuities across the interface and the slopes in the exterior region $\Omega_{1}$ are not properly captured. Increasing the parameter to $\beta=1000$ improves the solution, with small discontinuities and better approximation of the solution in the elements in the exterior domain. However the approximation in the cut elements is still far from the analytical solution. Again, the approximation with single enrichment is not able to represent the kinks of the solution on the interface.

Thus, it can be concluded that two Heaviside enrichments in cut elements are necessary to properly capture the solution in this example. The approximation with double enrichment 
can be easily handled in the X-HDG formulation, where continuity of the approximation between elements is imposed in weak form, whereas it is not straight-forward for a classical $\mathrm{X}$-FEM formulation.

3.6 Moving interface: circle collapsing at unit speed

The time-dependent heat equation

$$
\begin{aligned}
\frac{\partial u}{\partial t}-\nabla \cdot(\nu \nabla u)=f & \text { in } \Omega_{1}(t) \cup \Omega_{2}(t), \\
\llbracket u \boldsymbol{n} \rrbracket=\mathbf{0} & \text { on } \mathcal{I}(t), \\
\llbracket \nu \boldsymbol{n} \cdot \boldsymbol{\nabla} \rrbracket=0 & \text { on } \mathcal{I}(t), \\
u=u_{D} & \text { on } \Gamma_{D}=\partial \Omega, \\
u(x, 0)=u_{0}(x) & \text { in } \Omega_{1}(t) \cup \Omega_{2}(t),
\end{aligned}
$$

is solved over a square domain $\Omega=(-1,1)^{2}$ split into two subdomains, $\bar{\Omega}=\overline{\Omega_{1}} \cup \overline{\Omega_{2}}$, with an evolving circular interface $\mathcal{I}(t)$. The time-dependent radius is

$$
R(t)=0.8-t
$$

and material parameters are $\nu_{2}=5$ in $\Omega_{2}(t)=\left\{(x, y) \in \Omega \mid x^{2}+y^{2}<R(t)^{2}\right\}$ and $\nu_{1}=1$ in $\Omega_{1}=\Omega \backslash \overline{\Omega_{2}}$. Boundary conditions and source term are set such that the analytical solution is

$$
u(x, t)= \begin{cases}\frac{1}{\nu_{1}}\left(x^{2}+y^{2}\right)^{5 / 2} & \text { in } \Omega_{1}(t) \\ \frac{1}{\nu_{2}}\left(x^{2}+y^{2}\right)^{5 / 2}+\left(\frac{1}{\nu_{1}}-\frac{1}{\nu_{2}}\right) R(t)^{5} & \text { in } \Omega_{2}(t)\end{cases}
$$

The problem is discretized in time with a backward Euler method using a time step size $\Delta t$, leading to the local problems

$$
\begin{aligned}
& \left.\begin{array}{rl}
u^{n+1}+\Delta t \boldsymbol{\nabla} \cdot \boldsymbol{q}^{n+1}=u^{n}+\Delta t f^{n+1} & \text { in } K_{i} \\
\boldsymbol{q}^{n+1}+\nu \nabla u^{n+1}=0 & \text { in } K_{i} \\
u^{n+1}=\widehat{u}^{n+1} & \text { on } \partial K_{i}
\end{array}\right\} \text { if } \mathcal{I}^{n+1} \cap K_{i}=\emptyset \\
& u^{n+1}+\Delta t \boldsymbol{\nabla} \cdot \boldsymbol{q}^{n+1}=u^{n}+\Delta t f^{n+1} \quad \text { in } K_{i} \backslash \mathcal{I}^{n+1} \\
& \boldsymbol{q}^{n+1}+\nu \nabla u^{n+1}=0 \quad \text { in } K_{i} \backslash \mathcal{I}^{n+1} \\
& \left.\llbracket u^{n+1} \boldsymbol{n} \rrbracket=0 \quad \text { on } \mathcal{I}^{n+1} \cap K_{i}\right\} \text { if } \mathcal{I}^{n+1} \cap K_{i} \neq \emptyset \text {, } \\
& \llbracket \boldsymbol{q}^{n+1} \cdot \boldsymbol{n} \rrbracket=0 \\
& u^{n+1}=\widehat{u}^{n+1} \\
& \text { on } \mathcal{I}^{n+1} \cap K_{i} \\
& \text { on } \partial K_{i}
\end{aligned}
$$

where the superscript denotes the time instant. As usual, the solution at time $t^{n}$ is assumed to be known and the solution at time $t^{n+1}$ is to be computed in each time step. Following the same rationale as in sections 2.1 (for standard elements) and 2.2 (for cut elements) the local problems (22) are discretized leading to a local solver of the form (11)-(12) to express $u^{n+1}$ and $\boldsymbol{q}^{n+1}$ in terms of $\widehat{u}^{n+1}$, with a modified expression of the elemental matrices and vectors. The local equations closing the problem are exactly the same as for the steady case, that is (4) and (5), now evaluated at time $t^{n+1}$, with the corresponding discretizations in section 2.3, now involving $u^{n+1}, \boldsymbol{q}^{n+1}$ and $\widehat{u}^{n+1}$. Replacing the local solver in the global equations leads to a linear system involving only $\widehat{u}^{n+1}$ in every time step. 

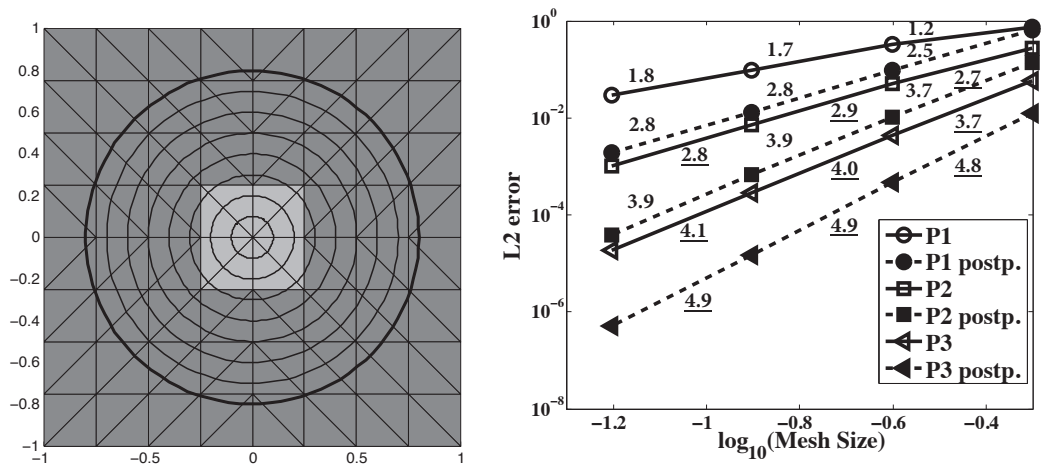

Fig. 22 Circle collapsing at unit speed: Interface location every 10 time steps with $\Delta t=0.01$ for $t \in[0,0.7]$ (left) and convergence history (right). Initial position is shown with a thicker line, gray elements are cut elements for $t=0.7$.

Similarly to the steady case, the X-HDG formulation differs from the standard HDG formulation only in the enriched approximation space for cut elements and cut faces, and the weak form of the local problem in cut elements. The weak form for the local problem in standard elements and for the global problem are the same as for standard HDG.

It is important noting that the enriched approximation spaces at time $t^{n}$ and time $t^{n+1}$ are not the same due to the fact that the interface location changes. Therefore, the Heaviside enrichment function is different. To facilitate the implementation of the discrete system, the solution $u^{n}$ is interpolated to the approximation space of time $t^{n+1}$ in every time step in this work, so that the enriched approximation space at time $t^{n+1}$ can be considered for the discretization of all terms in the equations.

The calculations are done on a sequence of four uniform meshes with changing approximation degree $k$. The time step size $\Delta t=0.01$ leads to a time error that is smaller than the spatial discretization errors. Figure 22 shows the location of the interface every ten time steps, for $t \in[0,0.7]$, and the $h$-convergence history. Optimal convergence rates are recorded for both the solution and the superconvergent solution, showing the applicability and good performance of X-HDG for problems with moving interfaces.

\section{Conclusions and final remarks}

The eXtended Hybridizable Discontinuous Galerkin (X-HDG) method is proposed for the solution of heat bimaterial problems. X-HDG is a new method to solve interface problems by combining the Hybridizable Discontinuous Galerkin (HDG) method and the philosophy of eXtended Finite Element Methods (X-FEM) together with an enriched approximation at the interface. The interface is represented as the 0 -level set of a function that is given by its nodal values on the computational mesh (usually the signed distance to the interface). The interface can cut through the elements in an arbitrary way and, therefore, the computational mesh does not need to align with the interface. The solution is enriched by Heaviside functions in the elements and faces cut by the interface, in order to properly represent the discontinuities in the derivatives of the solution, or even in the solution, across the interface.

The formulation for the global problem and for the local problem on elements not cut by the interface is the standard HDG formulation. For the elements cut by the interface, a 
new HDG local problem is derived taking into account the discontinuous approximation inside the element and the interface conditions to be imposed across the interface. Following the HDG ideas, a new trace variable on the interface is considered, which is locally eliminated afterwards using the interface conditions, leading to a local problem with the same structure as standard HDG. The main differences with standard HDG are (i) the modified local problem at cut elements and the corresponding matrices in the local solver, (ii) the modified numerical integration in cut elements and on cut faces, (iii) the increased number of degrees of freedom for the enriched approximation in cut elements and on cut faces, that has to be taken into account for the assembly of the matrices involving cut faces, (iv) a modified postprocessing for the superconvergent solution in cut elements. Thus, X-HDG keeps the structure and the computational efficiency of the HDG method, but without the need of fitting the mesh to the interface.

Numerical experiments also demonstrate that X-HDG keeps the HDG optimal convergence rates (of order $k+1$ in the $\mathcal{L}_{2}$ norm for the primal unknown and for its derivatives, and of order $k+2$ for the post-processed solution), with similar levels of accuracy.

A numerical example also shows the capability of X-HDG to handle elements that are split by the interface into more than two regions. In this situation, more than one enrichment function should be considered to ensure an independent approximation space in every region. With an X-HDG formulation different enrichment functions can be considered in each element, because continuity between elements is imposed in a weak form. This is not the case for X-FEM methods based on continuous approximations, for which using different enrichment spaces in each element is not possible or leads to a cumbersome implementation.

In the last numerical test, X-HDG is used to solve a transient problem, showing the potential of X-HDG for the solution of problems with evolving interfaces, avoiding continuous remeshing to fit the interface. The assessment of the efficiency of X-HDG for the solution of problems with moving interfaces with arbitrary shape, such as the Stefan problem, the comparison of different strategies for the projection of solutions between different enriched spaces in case of moving interfaces, and the extension of the formulation to bimaterial incompressible flow problems are subjects of ongoing work. The development of robust techniques to avoid ill-conditioning due to bad-cut elements in X-HDG is also an open issue.

Acknowledgements This work was supported by the DAFOH2 project (Ministerio de Economia y Competitividad, MTM2013-46313-R), the Erasmus Mundus Joint Doctorate SEED project (European Comission, 2013-1436/001-001-EMJD) and the Catalan goverment (Generalitat de Catalunya, 2009SGR875). The authors also acknowledge Ms. Esther Sala-Lardies (Universitat Politècnica de Catalunya) for letting them use her library for numerical integration in cut elements, and Prof. John E. Dolbow (Duke University) for the interesting discussions they had on the competitiveness of X-HDG in front of standard X-FEM.

\section{References}

1. Assêncio, D.C., Teran, J.M.: A second order virtual node algorithm for stokes flow problems with interfacial forces, discontinuous material properties and irregular domains. J. Comput. Phys. 250, 77 - 105 (2013)

2. Cheng, K.W., Fries, T.P.: Higher-order XFEM for curved strong and weak discontinuities. Int. J. Numer. Methods Eng. 82(5), 564-590 (2010)

3. Cockburn, B.: Discontinuous Galerkin methods for computational fluid dynamics. In: Encyclopedia of Computational Mechanics, vol. 3 (Fluids), chap. 4. Wiley, New York (2004)

4. Cockburn, B., Dong, B., Guzmán, J.: A superconvergent LDG-hybridizable Galerkin method for secondorder elliptic problems. Math. Comp. 77(264), 1887-1916 (2008) 
5. Cockburn, B., Gopalakrishnan, J., Lazarov, R.: Unified hybridization of discontinuous Galerkin, mixed, and continuous Galerkin methods for second order elliptic problems. SIAM J. Numer. Anal. 47(2), 1319-1365 (2009)

6. Cockburn, B., Gopalakrishnan, J., Nguyen, N.C., Peraire, J., Sayas, F.J.: Analysis of HDG methods for Stokes flow. Math. Comp. 80(274), 723-760 (2011)

7. Cockburn, B., Qiu, W., Shi, K.: Conditions for superconvergence of HDG methods for second-order elliptic problems. Math. Comp. 81(279), 1327-1353 (2012)

8. Dong, H., Wang, B., Xie, Z., Wang, L.L.: An unfitted hybridizable discontinuous Galerkin method for the poisson interface problem and its error analysis. Ima. J. Numer. Anal. (2016)

9. Dreau, K., Chevaugeon, N., Moës, N.: Studied X-FEM enrichment to handle material interfaces with higher order finite element. Comput. Methods Appl. Mech. Eng. 199(29-32), 1922-1936 (2010)

10. Fries, T.P.: A corrected XFEM approximation without problems in blending elements. Int. J. Numer. Methods Eng. 75(5), 503-532 (2008)

11. Fries, T.P., Belytschko, T.: The extended/generalized finite element method: An overview of the method and its applications. Int. J. Numer. Methods Eng. 84(3), 253-304 (2010)

12. Giorgiani, G., Fernández-Méndez, S., Huerta, A.: Hybridizable Discontinuous Galerkin p-adaptivity for wave propagation problems. Int. J. Numer. Methods Fluids 72(12), 1244-1262 (2013)

13. Giorgiani, G., Modesto, D., Fernández-Méndez, S., Huerta, A.: High-order continuous and discontinuous Galerkin methods for wave problems. Int. J. Numer. Methods Fluids 73(10), 883-903 (2013)

14. Gürkan, C., Sala-Lardies, E., Kronbichler, M. Fernández-Méndez, S.: eXtended hybridizable discontinous Galerkin (X-HDG) for void problems. J. Sci. Comput. 66(3), 1313-1333 (2015)

15. Hansbo, A., Hansbo, P.: An unfitted finite element method, based on Nitsche method for elliptic interface problems. Comput. Methods Appl. Mech. Eng. 191, 5537-5552 (2002)

16. Hesthaven, J., Warburton, T.: Nodal high-order methods on unstructured grids: I. Time-domain solution of Maxwell's equations. J. Comput. Phys. 181(1), 186-221 (2002)

17. Huerta, A., Angeloski, A., Roca, X., Peraire, J.: Efficiency of high-order elements for continuous and discontinuous Galerkin methods. Int. J. Numer. Methods Eng. 96(9), 529-560 (2013)

18. Huynh, L.T., Nguyen, N., Peraire, J., Khoo, B.: A high-order hybridizable discontinuous Galerkin method for elliptic interface problems. Int. J. Numer. Meth. Eng. 93(2), 183-200 (2013)

19. Kirby, R., Sherwin, S.J., Cockburn, B.: To CG or to HDG: A comparative study. J. Sci. Comput. 51(1), 183-212 (2011)

20. Legrain, G., Chevaugeon, N., Drau, K.: High order X-FEM and levelsets for complex microstructures: Uncoupling geometry and approximation. Comput. Methods Appl. Mech. Eng. 241244, 172 - 189 (2012)

21. Montlaur, A., Fernández-Méndez, S., Huerta, A.: Discontinuous Galerkin methods for the Stokes equations using divergence-free approximations. Int. J. Numer. Methods Fluids 57(9), 1071-1092 (2008)

22. Nguyen, N., Peraire, J., Cockburn, B.: A hybridizable discontinuous Galerkin method for Stokes flow. Comput. Methods Appl. Mech. Eng. 199(9-12), 582-597 (2010)

23. Nguyen, N.C., Peraire, J., Cockburn, B.: An implicit high-order hybridizable discontinuous Galerkin method for the incompressible Navier-Stokes equations. J. Comput. Phys. 230(4), 1147-1170 (2011)

24. Peraire, J., Persson, P.O.: The compact discontinuous Galerkin (CDG) method for elliptic problems. SIAM J. Sci. Comput. 30(4), 1806-1824 (2008)

25. Wang, B., Khoo, B.: Hybridizable discontinuous Galerkin method (HDG) for Stokes interface flow. J. Comput. Phys. 247, 262-278 (2013)

26. Yakovlev, S., Moxey, D., Kirby, R.M., Sherwin, S.J.: To CG or to HDG: A comparative study in 3D. J. Sci. Comput. 67(1), 192-220 (2015) 